\title{
Ultrafast Renormalization of the On-Site Coulomb Repulsion in a Cuprate Superconductor
}

\author{
Denitsa R. Baykusheva๑, ${ }^{1, *}$ Hoyoung Jang $\odot,{ }^{2}$ Ali A. Husain $\odot,{ }^{3,4,5}$ Sangjun Lee ${ }^{3,4}$ Sophia F. R. TenHuisen $\odot,{ }^{1,6}$ \\ Preston Zhou, ${ }^{1}$ Sunwook Park, ${ }^{7,8}$ Hoon Kim, ${ }^{7,8}$ Jin-Kwang Kim $\odot,{ }^{7,8}$ Hyeong-Do Kim $\odot,{ }^{2}$ Minseok Kim, ${ }^{2}$ \\ Sang-Youn Park $\odot,{ }^{2}$ Peter Abbamonte $\odot,{ }^{3,4}$ B. J. Kim, ${ }^{7,8}$ G. D. Gu, ${ }^{9}$ Yao Wang $\odot,{ }^{10, \dagger}$ and Matteo Mitrano $\oplus^{1, \ddagger}$ \\ ${ }^{1}$ Department of Physics, Harvard University, Cambridge, Massachusetts 02138, USA \\ ${ }^{2}$ PAL-XFEL, Pohang Accelerator Laboratory, Pohang, Gyeongbuk 37673, Republic of Korea \\ ${ }^{3}$ Department of Physics, University of Illinois at Urbana-Champaign, Urbana, Illinois 61801, USA \\ ${ }^{4}$ Materials Research Laboratory, University of Illinois at Urbana-Champaign, Urbana, Illinois 61801, USA \\ ${ }^{5}$ Quantum Matter Institute and Department of Physics and Astronomy, \\ University of British Columbia, Vancouver, British Columbia V6T 1Z4, Canada \\ ${ }^{6}$ John A. Paulson School of Engineering and Applied Sciences, \\ Harvard University, Cambridge, Massachusetts 02138, USA \\ ${ }^{7}$ Department of Physics, Pohang University of Science and Technology, Pohang 37673, South Korea \\ ${ }^{8}$ Center for Artificial Low Dimensional Electronic Systems, \\ Institute for Basic Science (IBS), 77 Cheongam-Ro, Pohang 37673, South Korea \\ ${ }^{9}$ Condensed Matter Physics and Materials Science Department, \\ Brookhaven National Laboratory (BNL), Upton, New York 11973 USA \\ ${ }^{10}$ Department of Physics and Astronomy, Clemson University, Clemson, South Carolina 29631, USA
}

(Received 25 June 2021; revised 19 September 2021; accepted 9 November 2021; published 20 January 2022)

Ultrafast lasers are an increasingly important tool to control and stabilize emergent phases in quantum materials. Among a variety of possible excitation protocols, a particularly intriguing route is the direct light engineering of microscopic electronic parameters, such as the electron hopping and the local Coulomb repulsion (Hubbard $U$ ). In this work, we use time-resolved x-ray absorption spectroscopy to demonstrate the light-induced renormalization of the Hubbard $U$ in a cuprate superconductor, $\mathrm{La}_{1.905} \mathrm{Ba}_{0.095} \mathrm{CuO}_{4}$. We show that intense femtosecond laser pulses induce a substantial redshift of the upper Hubbard band while leaving the Zhang-Rice singlet energy unaffected. By comparing the experimental data to time-dependent spectra of single- and three-band Hubbard models, we assign this effect to an approximately $140-\mathrm{meV}$ reduction of the on-site Coulomb repulsion on the copper sites. Our demonstration of a dynamical Hubbard $U$ renormalization in a copper oxide paves the way to a novel strategy for the manipulation of superconductivity and magnetism as well as to the realization of other long-range-ordered phases in light-driven quantum materials.

DOI: 10.1103/PhysRevX.12.011013

Subject Areas: Condensed Matter Physics

Strongly Correlated Materials

Superconductivity

\section{INTRODUCTION}

The electronic dynamics of strongly correlated materials are governed by a subtle competition between itinerancy due to hopping and localization driven by Coulomb repulsion $[1,2]$. The balance between these two tendencies is responsible for a wide variety of emergent quantum

\footnotetext{
*dbaykusheva@g.harvard.edu

†yaowang@g.clemson.edu

*mmitrano@g.harvard.edu
}

Published by the American Physical Society under the terms of the Creative Commons Attribution 4.0 International license. Further distribution of this work must maintain attribution to the author(s) and the published article's title, journal citation, and DOI. phases, and its manipulation through external perturbations is a central focus of modern condensed matter physics $[3,4]$. Ultrafast laser pulses offer an intriguing nonequilibrium control route, particularly when fields are strong enough (approximately $0.1-1 \mathrm{~V} / \AA$ ) to induce energy changes at the scale of the effective electronic interactions. Electronic hopping can be controlled by transiently bending the band structure (dynamical Franz-Keldysh effect) [5-10], by displacing atoms via nonlinear phonon excitation [11-13], or by coherent light-matter dressing of the band structure (Floquet engineering) [14-16]. However, manipulating the effective Coulomb repulsion with ultrafast lasers is less straightforward. Optical spectroscopy in organic solids hints at the possibility of modifying the effective on-site interaction (Hubbard $U$ ) by coherently driving local molecular vibrations [17-19] or enhancing 

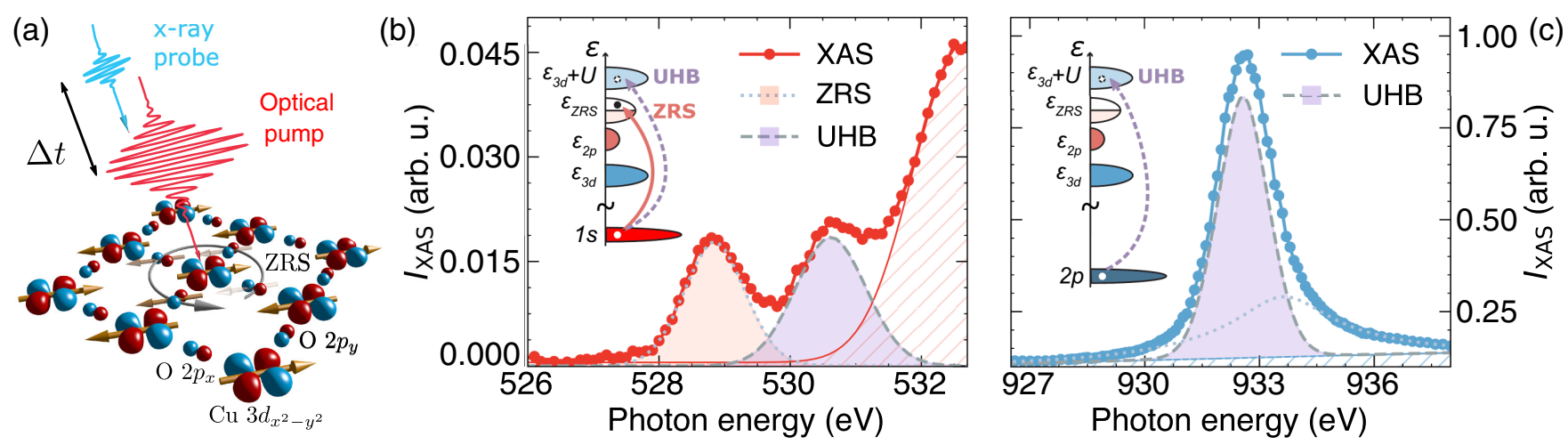

FIG. 1. Probing effective electronic interactions with x-ray absorption spectroscopy (XAS). (a) An LBCO $(x=9.5 \%)$ sample is driven by intense optical pump pulses at $1.55 \mathrm{eV}$, while its low-energy electronic structure is probed by exciting core electrons into unoccupied valence states with soft $\mathrm{x}$-ray pulses for variable time delay $\Delta t$. Equilibrium XAS spectrum at (b) $\mathrm{O} K$ and (c) $\mathrm{Cu} L_{3}$ edges, with transitions into Zhang-Rice singlets (ZRS) and upper Hubbard band (UHB) denoted by red and purple areas, respectively. Hatched areas indicate the main absorption edge, while the high-energy shoulder in (c) is a ligand hole sideband which we neglect in the rest of this work. A sketch of the density of states and the relevant x-ray transitions is reported within each panel.

intradimer hopping [20]. Yet, this route relies on the presence of molecular orbitals coupled to local structural degrees of freedom and cannot be readily extended to other classes of strongly correlated materials, like transition metal oxides. In these systems, the Hubbard $U$ is a strictly atomic property, and its modification requires alternative microscopic mechanisms, such as dynamical electronic screening [21-25] or Floquet-type dressing of the Coulomb repulsion [26]. Achieving dynamical tuning of the Hubbard $U$ in transition metal oxides would be particularly consequential for steering their multiple quantum phases, notably magnetism, multiferroicity, charge and spin order, and superconductivity $[27,28]$.

In the specific case of the high- $T_{c}$ cuprate superconductors, the local Coulomb repulsion has a pervasive effect on normal state properties as well as magnetic and superconducting phases $[29,30]$. The Hubbard $U$ increases the quasiparticle effective mass [31] and broadens the Fermi surface $[32,33]$. Furthermore, it sets the scale of the magnetic superexchange, and, in a spin-fluctuationmediated picture, it directly determines the superconducting pairing [34] and critical temperature [35]. Whether ultrafast lasers are able to modify the Hubbard $U$ in cuprate superconductors is an open experimental and theoretical problem.

Here, we demonstrate a pump-induced renormalization of the on-site Coulomb repulsion in $\mathrm{La}_{2-x} \mathrm{Ba}_{x} \mathrm{CuO}_{4}$ (LBCO), a single-layer cuprate with coexisting high-temperature superconductivity (up to $32 \mathrm{~K}$ ) and charge and spin order [36-38]. We directly probe the electronic density of states of LBCO by measuring its time-dependent $\mathrm{x}$-ray absorption spectrum and show that intense near-infrared pump pulses induce a significant transient shift of the absorption peaks. By mapping our data onto single- and three-band Hubbard models, we assign the shift to a renormalization of the local on-site Coulomb repulsion on the copper orbitals. Finally, we discuss implications of these results for the understanding of light-enhanced superconductivity, the generation of high harmonics in correlated materials, and the realization of coherent light-driven states.

\section{EXPERIMENTAL METHODS}

Time-resolved x-ray absorption spectroscopy (trXAS) is a valuable element-specific probe of the local electronic structure in light-driven materials [39-46], especially in insulating and/or poorly cleavable samples. This experiment makes use of the resonant soft x-ray scattering (RSXS) end station of the Pohang Accelerator Laboratory x-ray free electron laser (PAL-XFEL) [47]. We acquire trXAS spectra in fluorescence-yield mode at both the $\mathrm{O} K$ and $\mathrm{Cu} L_{3}$ edges with an energy resolution of 0.046 and $0.34 \mathrm{eV}$, respectively. The x-ray beam, focused to $120 \times 230 \mu \mathrm{m}^{2}$ and horizontally polarized, impinges at near-normal incidence and is detected by an avalanche photodiode at $2 \theta=150^{\circ}$. Shot-to-shot $\mathrm{x}$-ray intensity fluctuations are recorded with a gas-monitor detector and used to normalize the XAS signal. We excite the LBCO sample with 50-fs pulses centered at $1.55 \mathrm{eV}$, polarized in the $a b$ plane, and focused to $600 \times 600 \mu \mathrm{m}^{2}$ to obtain a fluence of $10 \mathrm{~mJ} / \mathrm{cm}^{2}$ (approximately $12 \mathrm{MV} / \mathrm{cm}$ peak electric field). Since the optical penetration depth $(370 \mathrm{~nm})$ exceeds that of the soft x-rays (170-290 nm), our trXAS spectra correspond to a homogeneously excited volume. The sample, a millimeter-sized single crystal of underdoped LBCO $\left(x=9.5 \%, T_{c}=32 \mathrm{~K}\right)$ [38], is cleaved to expose a fresh $a b$ surface and kept at $17 \mathrm{~K}$ throughout the entire experiment.

\section{EQUILIBRIUM X-RAY ABSORPTION SPECTRUM}

As shown in Fig. 1(a), the equilibrium electronic structure of the copper oxides is mainly determined by the in-plane $\mathrm{O}$ $2 p_{x, y}$ and $\mathrm{Cu} 3 d_{x^{2}-y^{2}}$ orbitals $[29,48]$. The $3 d$ orbitals form 

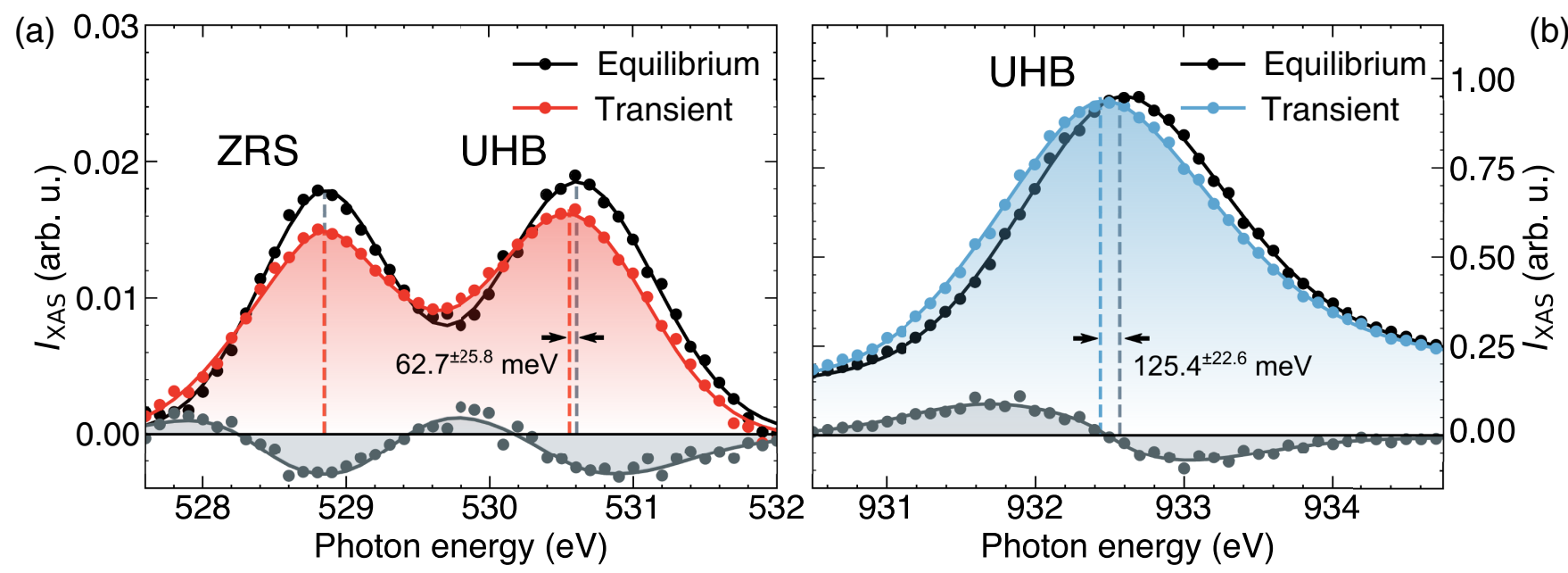

(b)

FIG. 2. Pump-induced reshaping of the X-ray absorption. XAS spectra before $(\Delta t \sim-1 \mathrm{ps})$ and after $(\Delta t \sim 0 \mathrm{ps})$ the pump arrival at both $\mathrm{O} K$ (a) and $\mathrm{Cu}_{3}$ (b) absorption edges. Symbols indicate equilibrium (black), transient (red and blue), and difference spectra (gray), while solid lines represent fits to the spectra. The main edge absorption in (a) is subtracted for clarity. The fit peak positions for each feature are marked by dashed lines. The UHB is found to shift by the energy indicated in the panels (error bars are $95 \%$ confidence intervals), while the ZRS position is unchanged.

two bands [lower and upper Hubbard bands (LHB and UHB, respectively)] due to the on-site Coulomb repulsion, with the LHB being pushed below the oxygen $2 p$ states. This band separation gives rise to a charge transfer (CT) gap, in which the lowest electronic transition occurs between oxygen (occupied) and copper (unoccupied) states. Upon hole doping, the density of states develops an additional feature at the lower end of the CT gap. This feature is generally attributed to a hybridized state between a local $3 d$ hole and a $2 p$ hole on the four surrounding oxygens and is known as the Zhang-Rice singlet (ZRS) [49,50]. In this work, we measure time-resolved absorption spectra at both the $\mathrm{O} K$ and $\mathrm{Cu} L_{3}$ edges to map the local density of states in the $\mathrm{CuO}_{2}$ planes and determine the transient effective interactions.

The equilibrium $\mathrm{O} K$ and $\mathrm{Cu} L_{3}$ edge absorption spectra are shown in Figs. 1(b) and 1(c). The $\mathrm{O} K$ spectrum features two pre-edge peaks at 528.9 and $530.5 \mathrm{eV}$ (see Sec. I in Supplemental Material [51] for further details). The lowerenergy transition involves ZRSs $\left(3 d^{9} \underline{L} \rightarrow \underline{1 s} 3 d^{9}\right.$, with $\underline{L}$ and $\underline{1 s}$ being, respectively, the $\mathrm{O} 2 p_{x, y}$ ligand and the $\mathrm{O} 1 s$ core holes) $[36,50,52]$. The higher-energy peak corresponds to a transition into the UHB $\left(3 d^{9} \rightarrow \underline{1 s} 3 d^{10}\right)$, which becomes dipole-allowed due to the mixing of $3 d^{10} \underline{L}$ and $3 d^{9}$ configurations in the ground state [53]. The $\mathrm{Cu} L_{3}$ edge spectrum in Fig. 1(c) instead exhibits a main peak at $932.6 \mathrm{eV}$ and a weaker shoulder at approximately $934 \mathrm{eV}$, which correspond to transitions into the UHB $\left(3 d^{9} \rightarrow \underline{2 p_{3 / 2}} 3 d^{10}\right)$ and into ligand states $\left(3 d^{9} \underline{L} \rightarrow \underline{2 p_{3 / 2}} 3 d^{10} \underline{L}\right)[53,54]$ (see Supplemental Material [51] Sec. I B for further details).

\section{TRANSIENT X-RAY ABSORPTION DYNAMICS}

Having assigned the spectral features at both absorption edges, we are now able to track light-induced changes of
LBCO's electronic structure. Figure 2 reports our key experimental observation. By comparing equilibrium and transient XAS spectra, we observe prompt photoinduced changes on both absorption edges. At the peak of the response, the UHB peak undergoes a substantial redshift, whereas the ZRS transition energy remains at its equilibrium value. The UHB shift is especially prominent at the $\mathrm{Cu} L_{3}$ edge, as its center energy decreases by $125 \mathrm{meV}$ compared to a $63-\mathrm{meV}$ shift at the $\mathrm{O} K$ edge. The integrated intensity of both peaks is unperturbed, and only the ZRS transition is found to broaden by approximately $20 \%$.

These photoinduced changes are prompt (close to the approximately 80-fs pump-probe cross-correlation) and relax to equilibrium following a single exponential behavior with a decay constant on the order of $100 \mathrm{fs}$ (see Fig. 3). These timescales are compatible with a relaxation process mediated by a dissipative bath of optical phonons [55] and in agreement with previous ultrafast optical spectroscopy [56-58], electron diffraction [59,60], and photoemission [61-65] experiments on multiple cuprate compounds.

Intriguingly, the UHB recovers more slowly than the ZRS peak. The UHB approaches equilibrium in $320 \mathrm{fs}$ (220 fs) at the $\mathrm{O} K\left(\mathrm{Cu} L_{3}\right)$ edge, while the ZRS decays over $100 \mathrm{fs}$ (see Supplemental Material [51], Sec. II B). This is consistent with holon-doublon recombination dynamics in MottHubbard and CT insulators, a mechanism also at play in other copper oxides [56,57,66-68], organic Mott insulators $[69,70]$, and cold atoms in optical lattices $[71,72]$. In our experiment, the $1.55-\mathrm{eV}$ pump excites electrons across the CT gap, thus creating holes (holons) and double occupancies (doublons) [73]. Unlike conduction carriers within the ZRS band, holon-doublon pairs need to dissipate an energy approximately equal to the CT gap in order to recombine. 

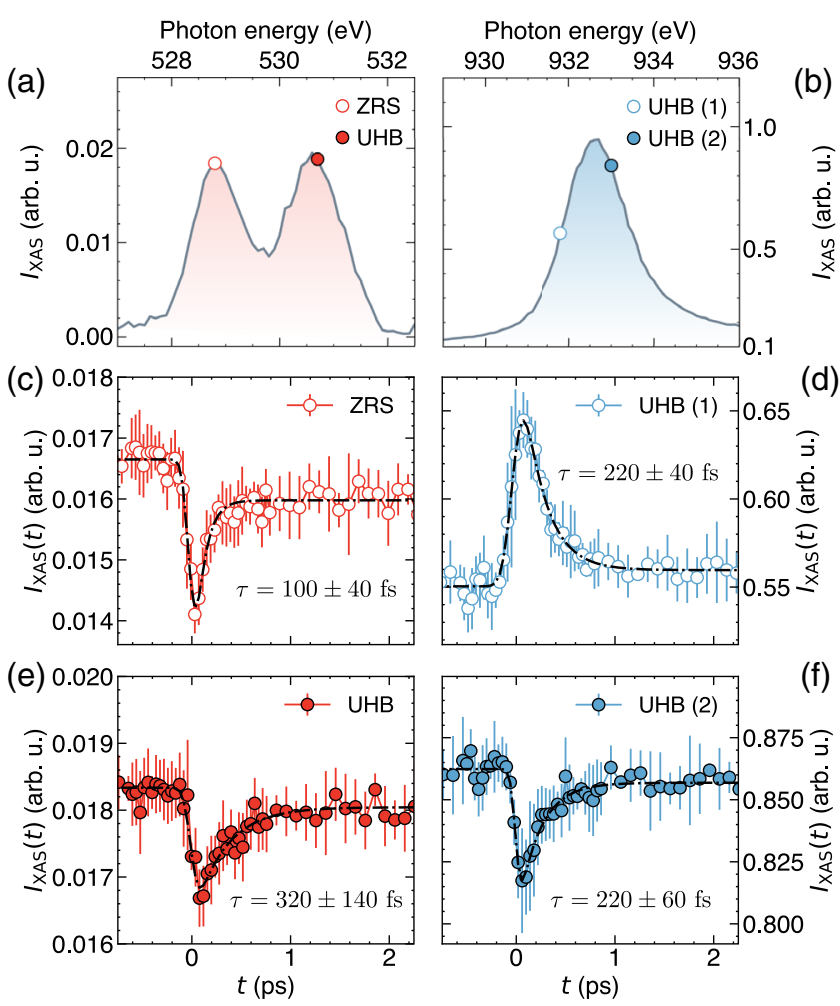

FIG. 3. Time dependence of the pump-induced electronic dynamics. Equilibrium XAS spectra at $\mathrm{O} K$ and $\mathrm{Cu} L_{3}$ absorption edges are shown in (a) and (b), respectively (shaded areas). Selected energies used to measure the time-dependent recovery are indicated by filled and empty symbols. (c) and (e) display the temporal evolution of ZRS and UHB peaks at the O $K$ edge, while points around the UHB peak at the $\mathrm{Cu} L_{3}$ edge are reported in (d) and (f). Error bars on the symbols represent standard deviations, while dash-dotted lines are fit to the data (see Supplemental Material [51] Sec. II B for further details).

This process generally requires a large number of lowerenergy scattering partners (e.g., phonons, magnons, or lower-energy charge fluctuations), thus making decay events increasingly rare $[66,72,73]$. As a consequence, the UHB relaxation rate gets exponentially suppressed with the increasing CT gap $[66,73]$ and becomes slower than the conduction-band quasiparticle relaxation.

These data cannot be rationalized in terms of pumpinduced doping or heating effects. Hole doping in the closely related $\mathrm{La}_{2-x} \mathrm{Sr}_{x} \mathrm{CuO}_{4}$ [52-54] leads to significant intensity changes and an increased energy separation between UHB and ZRS transitions, while we observe a decrease in the energy gap between the two peaks. Electronic heating redshifts the entire absorption spectrum through the creation of unoccupied states at lower energies [40,74], with the ZRS shifting more (129 meV) than the UHB peak $(87 \mathrm{meV})$ at an estimated $T_{e} \sim 2500 \mathrm{~K}$ (see the Appendix C and Sec. III B of Supplemental Material [51] for electronic temperatures between $T_{e}=0$ and $T_{e}=5300 \mathrm{~K}$ ). In contrast, we experimentally observe a shift of the UHB while the ZRS remains fixed. Finally, the excitation of optical or acoustic phonons $[60,61,75]$, which may form multiplet structures [76] and broaden the XAS spectrum beyond the natural core-hole width $[77,78]$, primarily affects the intensity and width (and not the energy) of the near-edge transitions. Therefore, a more natural explanation for our data is a dynamic renormalization of the effective electronic interactions.

\section{QUANTIFYING THE TRANSIENT HUBBARD $U$}

To determine which electronic interactions are affected by the pump, we calculate the transient $\mathrm{x}$-ray absorption of a three-band Hubbard model with renormalized electronic parameters [77,79-82]. The calculation is performed at $12.5 \%$ hole doping in order to reduce the computational complexity (see Appendix A for further details). While the superconducting and charge-ordered phases at $12.5 \%$ hole doping are distinct from the ones of our $x=9.5 \%$ sample [38], the XAS spectra are similar enough in intensity and peak energy [52,53] to justify this simplification. The theoretical spectra closely resemble the experimental $\mathrm{x}$-ray absorption in Figs. 1 and 2. The $\mathrm{O} K$ edge spectrum in Fig. 4(a) features one prominent ZRS peak approximately $1 \mathrm{eV}$ below the edge threshold ( $\left.E_{\text {edge }}\right)$ and additional spectral peaks around $1 \mathrm{eV}$ which correspond to the UHB. The $\mathrm{Cu} L_{3}$ edge spectrum is instead characterized by a prominent UHB peak around $E_{\text {edge }}$ and a weaker peak $1.2 \mathrm{eV}$ higher corresponding to the $3 d^{9} \underline{L}$ states. All spectra are broadened only by accounting for the core-hole lifetime, which is assumed to be $0.2 \mathrm{eV}$ at both the $\mathrm{O} K$ and 

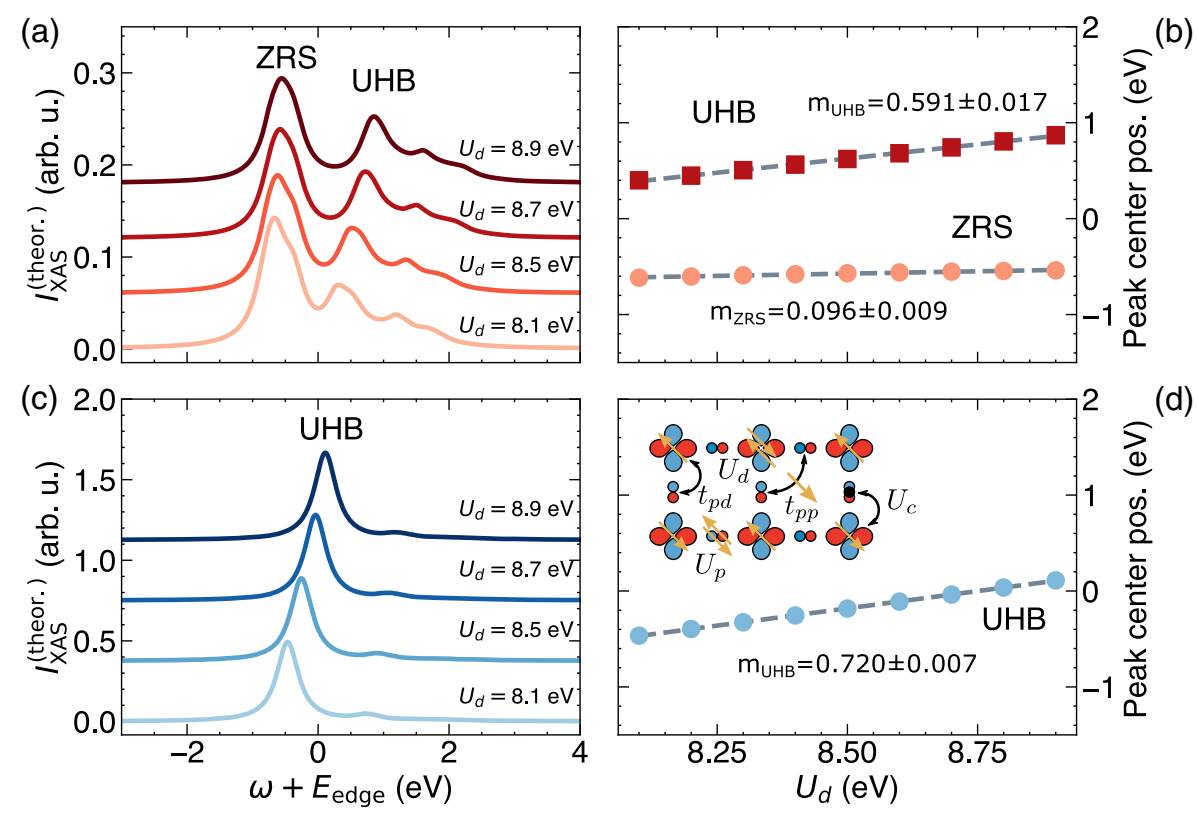

FIG. 4. Theoretical XAS spectra of a three-band Hubbard model for variable on-site Coulomb repulsion $U_{d}$. (a) and (c) show XAS spectra at the oxygen and copper absorption edges, respectively. The value of $U_{d}$ used in the calculation is reported near each curve, while all the other electronic parameters are kept fixed at their equilibrium values. (b) and (d) show UHB and ZRS center positions, respectively, as a function of $U_{d}$, together with their fitted slopes. The inset in (d) is a cartoon of the three-band Hubbard system used in this calculation with hopping amplitudes $t_{p d}$ and $t_{p p}$, on-site Coulomb repulsions $U_{d}$ and $U_{p}$, and core-hole interaction $U_{c}$.

$\mathrm{Cu} L_{3}$ edges [83,84]. At the $\mathrm{O} K$ edge, the separation between the ZRS and UHB centroids for $U_{d}=8.5 \mathrm{eV}$ matches the experimental separation (approximately $1.8 \mathrm{eV}$ ), while the overall UHB intensity is about $85 \%$ the one of the ZRS (as expected for the $12.5 \%$ hole-doped spectrum [52]). To accurately determine the slope of the UHB peak shift, we track only the sharpest feature, which is located at slightly lower energy. We systematically tune each parameter of the three-band Hubbard Hamiltonian and map the corresponding effect on a static XAS spectrum capturing the excited steady state. Our observation of a redshifted UHB and fixed ZRS transitions is reproduced only by changing the on-site Coulomb repulsion $U_{d}$ [see Figs. 4(a) and 4(c)], i.e., the energy cost to double occupy a $\mathrm{Cu}$ orbital or, equivalently, the energy of the charge fluctuation $d_{i}^{9} d_{j}^{9} \rightarrow d_{i}^{8} d_{j}^{10}(i$ and $j$ denoting different lattice sites). All other parameters $\left(t_{p p}, U_{p}\right.$, and $t_{p d}$ ) introduce an energy shift of both peaks in tandem (see Supplemental Material [51], Sec. III). The shift of the sole UHB band crucially identifies the photoinduced dynamics as a transient renormalization of the Hubbard $U$ on the copper sites. By fitting the theoretical spectra (see Supplemental Material [51], Sec. III), we subsequently convert the peak shift into an absolute change $\delta U_{d}$ of the Hubbard $U$. The UHB peak position is found to shift linearly for small $\delta U_{d}$ [see Figs. 4(b) and 4(d)], while the ZRS is almost unaffected. The slope of the UHB position as a function of $U_{d}$ at the $\mathrm{Cu} L_{3}$ edge is larger than that at the $\mathrm{O} K$ edge, consistent with the experiment, and the observed shifts are reproduced by a Hubbard $U$ reduction $\delta U_{d} \sim 140 \mathrm{meV}$.

\section{A MINIMAL THEORETICAL DESCRIPTION}

While providing quantitative information about the electronic interactions, it is not obvious a priori that the transient dynamics can be fully captured by a quasiequilibrium three-band Hubbard model with tuned electronic parameters. Therefore, we perform a full time-dependent exact diagonalization calculation of the light-driven $\mathrm{x}$-ray absorption spectrum. To do this, we downfold the threeband Hamiltonian to an effective 2D, single-band Hubbard model $[49,77]$. In this transition, the three-band UHB maintains its original character, while the three-band ZRS band becomes the single-band LHB. Nonbonding and Zhang-Rice triplet states from the three-band model are ignored, and the single-band Mott gap is identified with the three-band CT gap $[85,86]$. We include the pump pulse through the standard Peierls substitution and calculate the XAS spectra as a function of the pump-probe time delay, as shown in Fig. 5. The calculations are performed on a 12D Betts cluster with two holes in order to preserve SU(2) symmetry (see Appendix B for further details). At equilibrium, the XAS signal features two distinct transitions into the LHB and UHB, qualitatively agreeing with the experimental $\mathrm{O} K$ edge spectra. The peak width is determined by two contributions: the intrinsic core-hole lifetime (set to $0.15 \mathrm{eV}$ ) and an extrinsic energy broadening introduced by the finite probe pulse (see Appendix B). The 
(a)

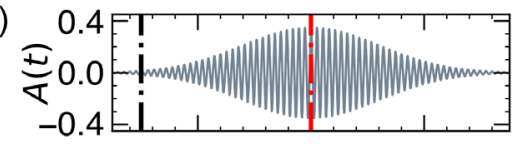

(b)
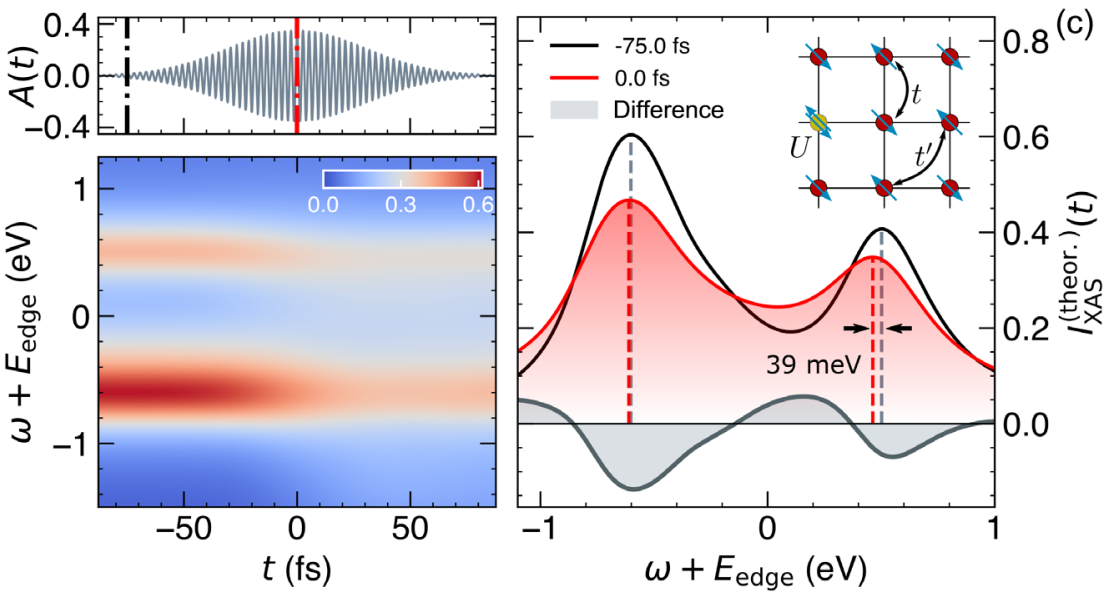

FIG. 5. Transient XAS spectrum of the single-band Hubbard model in two dimensions. (a) Time-dependent vector potential of the pump pulse used in the time-dependent calculations. Colored dashed lines indicate selected time delays for the snapshots in (c). (b) Full trXAS spectrum simulation as a function of the time delay and photon energy. (c) trXAS spectra at different pump-probe delays before (black) and during the pump (red). The peak positions of the UHB features are marked by dashed lines. The inset depicts the single-band Hubbard model with $t, t^{\prime}$ hopping amplitudes and on-site Coulomb repulsion $U$.

UHB peak intensity is about $70 \%$ of the LHB peak intensity [as opposed to the equally intense peaks in Fig. 2(a)], while the energy separation is $U-U_{c} \sim 1 \mathrm{eV}$ [87] with $U_{c}$ being the core-hole attractive interaction. When the pump and probe pulses overlap, the peak distance shrinks by approximately $40 \mathrm{meV}$, thus indicating a transient reduction of the Hubbard $U$ by $4 \%$. This Mott gap closure is quantitatively consistent with the $3 \%$ CT gap reduction visible in Fig. 2(a). The spectra also exhibit a general broadening and an increase of in-gap spectral weight, likely due to the creation of particle-hole excitations. The UHB shift is found to increase nonlinearly with the pump electric field, in agreement with our experimental findings (see Appendix D). Our dynamical single-band calculation captures the experimental UHB peak shift at a quantitative level and corroborates the three-band picture of a pumprenormalized on-site Coulomb repulsion.

\section{POSSIBLE MICROSCOPIC MECHANISMS}

We now discuss the possible microscopic origin of the observed Hubbard $U$ reduction. A first scenario involves the dynamical enhancement of dielectric screening $[21,23,24]$. The pump pulse promotes electrons from localized levels into highly delocalized states, which increases the screening of the local Coulomb interaction. In a multiband model, screening arises from both interband and intraband excitations, while second-order (of order $t^{2} / U$ ) doublon-holon and doublon-doublon interactions are the dominant screening modes in a single-band system.

The presence of second-order charge and spin fluctuations is compatible with the scattering from antiferromagnetic spin fluctuations observed in ultrafast reflectivity experiments [58]. Intriguingly, this mechanism leads to an average dynamical reduction of the Hubbard $U$ on both $p$ and $d$ orbitals [21,23,24], while the fixed ZRS energy hints at an unperturbed Coulomb repulsion on the $p$ orbitals. Furthermore, a transient enhancement of dielectric screening, being proportional to the intensity instead of the electric field, would be accompanied by oscillations of the UHB position at twice the frequency of the laser field (beyond our current time resolution) [21,24].

A second scenario involves instead a Floquet-type dressing of the effective electronic interactions $[26,88]$. The vector potential of the pump dynamically alters the Hubbard $U$ through both the average doublon number and their mutual interactions (of order $t^{2} / U$ ). Notably, this scheme could lead to either an increase or a decrease of the Hubbard $U$ depending on the pump field strength. Based on the observation that the Hubbard $U$ renormalization lifetime is longer than the pump pulse duration (see Fig. 3), we argue that dynamical screening may be dominant over Floquet dressing. Importantly, while the observation of a dynamical Hubbard $U$ does not depend on the validity of a specific microscopic scenario, future experiments with attosecond $\mathrm{x}$-ray pulses resolving coherent dynamics at the $U$ energy scale $[89,90]$ will be able to distinguish between these two microscopic mechanisms.

\section{CONCLUSION}

In summary, our experiment provides clear spectroscopic evidence for a transient and reversible renormalization of the Hubbard $U$ in a cuprate superconductor. This result has broad implications for photoinduced phase transitions and nonlinear phenomena in strongly correlated materials.

First, it provides a possible key to interpret the recent observation of light-enhanced superconductivity in cuprates at high excitation densities [91-95], which still lacks a comprehensive microscopic understanding. A $140-\mathrm{meV}$ 
reduction of the Hubbard $U$ will close the CT gap by $70 \mathrm{meV}$, or $3 \%$ of its equilibrium value (in agreement with recent ultrafast optical experiments on $\mathrm{YBa}_{2} \mathrm{Cu}_{3} \mathrm{O}_{7-\delta}$ [96]). Since the CT gap magnitude anticorrelates with the equilibrium superconducting $T_{c}$ in both theory and experiments [97,98], such reduction might be favorable to the onset of out-ofequilibrium pairing. Moreover, in a spin-fluctuation-mediated picture, the $\mathrm{CT}$ gap closure will be accompanied by a renormalization of the pairing interaction and of the spin fluctuation spectrum $[34,99]$. A lower CT gap will also increase the exchange interaction and, consequently, the characteristic spin fluctuation energy [100]. These effects might quantitatively explain the enhancement of superconducting correlations in photoexcited $\mathrm{La}_{2-x} \mathrm{Ba}_{x} \mathrm{CuO}_{4}$ $[93,94]$ and could be resolved in future time-resolved resonant inelastic $\mathrm{x}$-ray scattering experiments $[101,102]$.

A dynamical renormalization of the band structure could also represent an essential ingredient to boost the highharmonic generation efficiency in correlated materials, as recently discussed in the case of $\mathrm{NiO}[10,21]$. A lower Mott gap will indeed reduce the energy cost of Zener tunneling and multiphoton ionization processes, thus promoting electron excitation and harmonic emission under intense light irradiation.

Finally, achieving on-demand manipulation of the Coulomb repulsion in correlated materials may enable the observation of novel nonequilibrium states of matter, such as fragile quantum spin liquids in frustrated magnets [103-109] and $\eta$-paired superconductivity in driven Mott insulators [110-113]. In the former, a dynamical Hubbard $U$ could modify the exchange interactions and induce a transition from an antiferromagnet to an entangled spin liquid state. In the latter, tuning the Hubbard $U$ in a drivendissipative Mott insulator will dynamically alter the doublon number [111] and the energy spectrum of the driven Hamiltonian [110,114], thus paving the way to the onset of long-range staggered superconducting correlations.

\section{ACKNOWLEDGMENTS}

We thank M. Buzzi, A. Cavalleri, M. P. M. Dean, T. P. Devereaux, M. Eckstein, D. Golež, M. Li, B. Moritz, D. Nicoletti, A. H. Reid, A. Rubio, G. Sawatzky, and M. Sentef for insightful discussions. M. M. was supported by the William F. Milton Fund at Harvard University. D. R. B. acknowledges support from the Swiss National Science Foundation through Project No. P400P2_194343. B. J. K. was supported by IBS-R014-A2. H. J. acknowledges support by the National Research Foundation grant funded by the Korea government (MSIT) (Grant No. 2019R1F1A1060295). S. L., A. A. H., and P. A. acknowledge support from Department of Energy (DOE) Grant No. DE-FG02-06ER46285. P. A. acknowledges support from the Gordon and Betty Moore Foundation EPiQS Initiative through Grant No. GBMF9452. A. A. H. acknowledges support from the Max Planck-UBC-UTokyo
Center for Quantum Materials and the Canada First Research Excellence Fund, Quantum Materials and Future Technologies Program. The trXAS experiments were performed at the SSS-RSXS end station (Proposal No. 2020-2nd-SSS-006) of the PAL-XFEL funded by the Korea government (MSIT). Work at Brookhaven National Laboratory was supported by the U.S. DOE, Office of Science, Office of Basic Energy Sciences, under Contract No. DESC0012704. This research used resources of the National Energy Research Scientific Computing Center (NERSC), a U.S. Department of Energy Office of Science User Facility operated under Contract No. DE-AC02$05 \mathrm{CH} 11231$.

\section{APPENDIX A: CALCULATING THE XAS SPECTRUM OF A THREE-BAND HUBBARD MODEL}

In this work, we perform exact diagonalization calculations of the XAS spectrum of a three-orbital Hubbard model on a $2 \mathrm{D} \mathrm{Cu}_{8} \mathrm{O}_{16}$ cluster for two different doping levels: $0 \%$ (half filling) and $12.5 \%$ (underdoped). The threeorbital Hubbard Hamiltonian considered here is [77,79-82]

$$
\begin{aligned}
\hat{\mathcal{H}}_{v}= & -\sum_{\langle i, j\rangle \alpha \sigma} t_{p d}^{i j}\left(d_{i \sigma}^{\dagger} p_{j \alpha \sigma}+\text { H.c. }\right) \\
& -\sum_{\left\langle j, j^{\prime}\right\rangle \sigma} t_{p j}^{j j^{\prime}}\left(p_{j \alpha \sigma}^{\dagger} p_{j^{\prime} y \sigma}+\text { H.c. }\right)+\sum_{i \sigma} \epsilon_{d} n_{i \sigma}^{d}+\sum_{j \alpha \sigma} \epsilon_{p} n_{j \alpha \sigma}^{p} \\
& +\sum_{i} U_{d} n_{i \uparrow}^{d} n_{i \downarrow}^{d}+\sum_{j \alpha} U_{p} n_{j \alpha \uparrow}^{p} n_{j \alpha \downarrow}^{p} .
\end{aligned}
$$

Here, $d_{i \sigma}^{\dagger}\left(p_{j \alpha \sigma}^{\dagger}\right)$ creates a hole with spin $\sigma$ at a $\mathrm{Cu}$ site $i$ (O site $j$ ), and $n_{i \sigma}^{d}\left(n_{j \sigma}^{p}\right)$ is the $\mathrm{Cu}(\mathrm{O})$ hole number. The subindex $\alpha=\{x, y\}$ labels the symmetry of the $2 p_{x, y}$ orbital. The first two terms in Eq. (A1) are in-plane hopping terms with amplitudes $t_{p d}$ and $t_{p p}$, and the next two represent the charge-transfer energy (difference of the on-site energies $\Delta_{\mathrm{ct}}=\epsilon_{p}-\epsilon_{d}$ ), while the last two terms are the on-site Hubbard interactions. At equilibrium, $\epsilon_{p}-\epsilon_{d}=$ $3.23 \mathrm{eV}, U_{d}=8.5 \mathrm{eV}, U_{p}=4.1 \mathrm{eV},\left|t_{p d}\right|=1.13 \mathrm{eV}$, and $\left|t_{p p}\right|=0.49 \mathrm{eV}$. The intermediate core-hole state is described by the additional Hamiltonian operator

$$
\hat{\mathcal{H}}_{c}=\sum_{i \sigma} E_{\text {edge }}\left(1-n_{i \sigma}^{c}\right)-U_{c} \sum_{i \sigma \sigma^{\prime}} n_{i \sigma}^{v}\left(1-n_{i \sigma^{\prime}}^{c}\right),
$$

where $E_{\text {edge }}$ is the edge energy ( $E_{\text {edge }}=938 \mathrm{eV}$ for $\mathrm{Cu}$ and $E_{\text {edge }}=530 \mathrm{eV}$ for O atoms), $n_{i \sigma}^{c, v}$ the core (c) or valence $(v)$ electron number for the site corresponding to the specific edge (i.e., at $\mathrm{Cu} 2 p$ orbital for $\mathrm{Cu} L_{3}$ edge and at $\mathrm{O} 1 s$ orbital for $\mathrm{O} K$ edge), and $U_{c}$ the core-hole potential ( 9 and $6 \mathrm{eV}$ for $\mathrm{Cu}$ and $\mathrm{O}$, respectively). At the $\mathrm{Cu} L_{3}$ edge, we include an additional spin-orbit coupling term $\lambda \sum_{i \sigma \sigma^{\prime} \alpha \alpha^{\prime}} p_{i \alpha \sigma}^{\dagger} \chi_{\alpha \alpha^{\prime}}^{\sigma \sigma^{\prime}} p_{i \alpha^{\prime} \sigma^{\prime}}$ with $\lambda=13 \mathrm{eV}$. In this case, $\chi_{\alpha \alpha^{\prime}}^{\sigma \sigma^{\prime}} \equiv\left\langle p_{\alpha \sigma}|\boldsymbol{l} \cdot \boldsymbol{s}| p_{\alpha^{\prime} \sigma^{\prime}}\right\rangle$ is 
the spin-orbit coupling coefficient. The XAS spectrum is then

$$
\begin{aligned}
I_{\mathrm{XAS}}(\omega, T)= & -\sum_{n} \frac{e^{-E_{n} / k_{B} T}}{\pi Z} \\
& \times \operatorname{Im}\left\langle\psi_{n}\left|\mathcal{D}^{\dagger} \frac{1}{\omega+E_{n}-\mathcal{H}+i \delta} \mathcal{D}\right| \psi_{n}\right\rangle,
\end{aligned}
$$

where $Z=\sum_{n} e^{-E_{n} / k_{B} T}$ is the partition function with $k_{B}$ denoting the Boltzmann constant, $\left|\psi_{n}\right\rangle$ is the $n$th eigenstate of the (Hubbard) Hamiltonian with the eigenenergy $E_{n}$, and $\delta$ is the broadening corresponding to the inverse lifetime of the intermediate state (core hole). Unless otherwise specified, XAS spectra are calculated at zero temperature. Finally, the dipole operators $\mathcal{D}$ define the transformation between the core and the valence level selected by the x-ray absorption edge under the sudden approximation and take the form

$$
\begin{aligned}
\mathcal{D}_{\mathrm{O}-K} & =A_{\mathrm{O}-K} \sum_{\sigma} c_{j \sigma}^{p_{x}^{\dagger}} c_{j \sigma}^{c}, \\
\mathcal{D}_{\mathrm{Cu}-L_{3}} & =A_{\mathrm{Cu}-L_{3}} \sum_{\sigma} c_{i \sigma}^{d \dagger} c_{i \sigma}^{c} .
\end{aligned}
$$

Since we do not compare absolute intensities among different edges, we ignore both the matrix elements $A_{\mathrm{O}-K}$ and $A_{\mathrm{Cu}-L_{3}}$.

\section{APPENDIX B: TIME-RESOLVED XAS SPECTRUM OF A DRIVEN SINGLE-BAND HUBBARD MODEL}

The calculation of the trXAS spectrum following a strong pump excitation is performed by first downfolding the three-band Hamiltonian defined in Eqs. (A1) and (A2) to a single-band 2D Hubbard model (complemented by an additional attractive core-hole potential term $U_{c}$ ):

$$
\begin{aligned}
\hat{\mathcal{H}}_{0}= & -\sum_{\langle i, j\rangle \sigma} t_{h}^{i j}\left(d_{j \sigma}^{\dagger} d_{i \sigma}+\text { H.c. }\right)+U \sum_{i} n_{i \uparrow}^{(d)} n_{i \downarrow}^{(d)} \\
& +E_{\text {edge }} \sum_{i \alpha \sigma}\left(1-n_{i \alpha \sigma}^{(p)}\right)-U_{c} \sum_{i \alpha \sigma \sigma^{\prime}} n_{i \sigma}^{(d)}\left(1-n_{i \alpha \sigma^{\prime}}^{(p)}\right) \\
& +\lambda \sum_{i \alpha \alpha^{\prime} \sigma \sigma^{\prime}} p_{i \alpha \sigma}^{\dagger} \chi_{\alpha \alpha^{\prime}}^{\sigma \sigma^{\prime}} p_{i \alpha^{\prime} \sigma^{\prime}},
\end{aligned}
$$

with $U$ being the on-site Coulomb interaction and $t_{h}^{i j}$ the hopping integral between sites $i$ and $j . d_{i \sigma}\left(d_{i \sigma}^{\dagger}\right)$ annihilates (creates) a $3 d_{x^{2}-y^{2}}$ electron at site $i$ with spin $\sigma$, whereas $p_{i \alpha \sigma}\left(p_{i \alpha \sigma}^{\dagger}\right)$ annihilates (creates) an electron in the $2 p_{\alpha}$ orbital $(\alpha=x, y) \cdot n_{i}^{(d)}=d_{i} d_{i}^{\dagger}$ is the core-hole number operator. We set the nearest-neighbor hopping amplitude to $t_{h}=0.3 \mathrm{eV}$, the next-nearest hopping to $t_{h}^{\prime}=-0.09 \mathrm{eV}$, and the on-site repulsion to $U=2.4 \mathrm{eV}$ and fix the core-hole potential to $U_{c}=0.9 \mathrm{eV}$. The light-matter interaction with the pump vector potential $\mathbf{A}(t)$ is included via the standard Peierls substitution: $c_{i \sigma} \rightarrow c_{i \sigma} \exp \left[-i \int_{-\infty}^{\mathbf{r}_{i}} \mathbf{A}\left(\mathbf{r}^{\prime}, t\right) \cdot d \mathbf{r}^{\prime}\right]$, where $c_{i \sigma}\left(c_{i \sigma}^{\dagger}\right)$ is the annihilation (creation) operator for an electron with spin $\sigma$ on site $i$. The pump is a Gaussian pulse

$$
\mathbf{A}(t)=A_{0} e^{-t^{2} / \sigma_{t}^{2}} \cos (\Omega t) \hat{\mathbf{e}},
$$

with $\Omega=1.55 \mathrm{eV}$ energy (closely matched to the experimental conditions) and polarized as $\hat{\mathbf{e}}=(1 / \sqrt{2}, 1 / \sqrt{2})$ (linear in the $a b$ plane). The width of the pulse is set to $13.7 t_{h}^{-1}$, or approximately $71 \mathrm{fs}$ at full width half maximum (in the electric field), and the peak amplitude of the vector potential is set to $A_{0}=0.35$ (corresponding to a peak electric field of $12.3 \mathrm{MV} / \mathrm{cm}$ ). The trXAS spectrum is then evaluated by calculating the two-time correlation function [115]:

$$
\begin{aligned}
\mathcal{B}(\omega, t)= & \iint_{-\infty}^{+\infty} g\left(t_{1} ; t\right) g\left(t_{2} ; t\right) e^{i \omega\left(t_{2}-t_{1}\right)} e^{-\left|t_{2}-t_{1}\right| / \tau_{\text {core }}} \\
& \times\left\langle\hat{\mathcal{D}}_{\mathbf{q} \varepsilon}^{\dagger}\left(t_{2}\right) \hat{\mathcal{D}}_{\mathbf{q} \varepsilon}\left(t_{1}\right)\right\rangle_{0} d t_{1} d t_{2} .
\end{aligned}
$$

Here, $\hat{\mathcal{D}}_{\mathbf{q} \varepsilon}(t)=\sum_{i \sigma} e^{-i \mathbf{q} \cdot \mathbf{r}_{i}}\left(A_{\varepsilon} d_{i \sigma}^{\dagger} p_{i \sigma}+\right.$ H.c. $)$ is the dipole operator and $A_{\varepsilon}$ the dipole matrix element of the corresponding transition for a photon with polarization state $\boldsymbol{\varepsilon}$, which is set to unity in this paper. The core-hole lifetime is set to be $0.15 \mathrm{eV}$ in our simulation. The linearly polarized (along $x$ ) probe pulse is represented by a Gaussian pulse $g(\tau, t)=e^{-(t-\tau)^{2} /\left(2 \sigma_{\mathrm{pr}}\right)^{2}} / \sqrt{2 \pi} \sigma_{\mathrm{pr}}$ of pulse width $\sigma_{\mathrm{pr}}=$ $13.7 t_{h}^{-1}$ (30 fs). $\mathbf{q}$ denotes the momentum transfer between incident and scattered photons, which is zero for trXAS (cf. discussion in Ref. [115]). The calculated trXAS spectrum shown in Fig. 5 is centered at the $\mathrm{Cu} L_{3}$ edge with $E_{\text {edge }}=938 \mathrm{eV}$ (bare edge energy without spinorbit splitting), and the peak energy at each delay is extracted by determining the maximum of each spectral feature.

\section{APPENDIX C: CALCULATION OF POSSIBLE PUMP-INDUCED HEATING EFFECTS ON THE X-RAY ABSORPTION SPECTRUM}

In this appendix, we consider whether the transient reshaping of the experimental x-ray absorption spectrum could be due to pump-induced sample heating. We first determine the maximum electronic temperature increase expected under the experimental pump excitation conditions and then perform an exact diagonalization calculation of the theoretical XAS spectrum of a three-band Hubbard model at such electronic temperature value. For the first step, we follow the procedure described in the Supplemental Material of Ref. [95] and assume the following form for the specific heat of LBCO: 

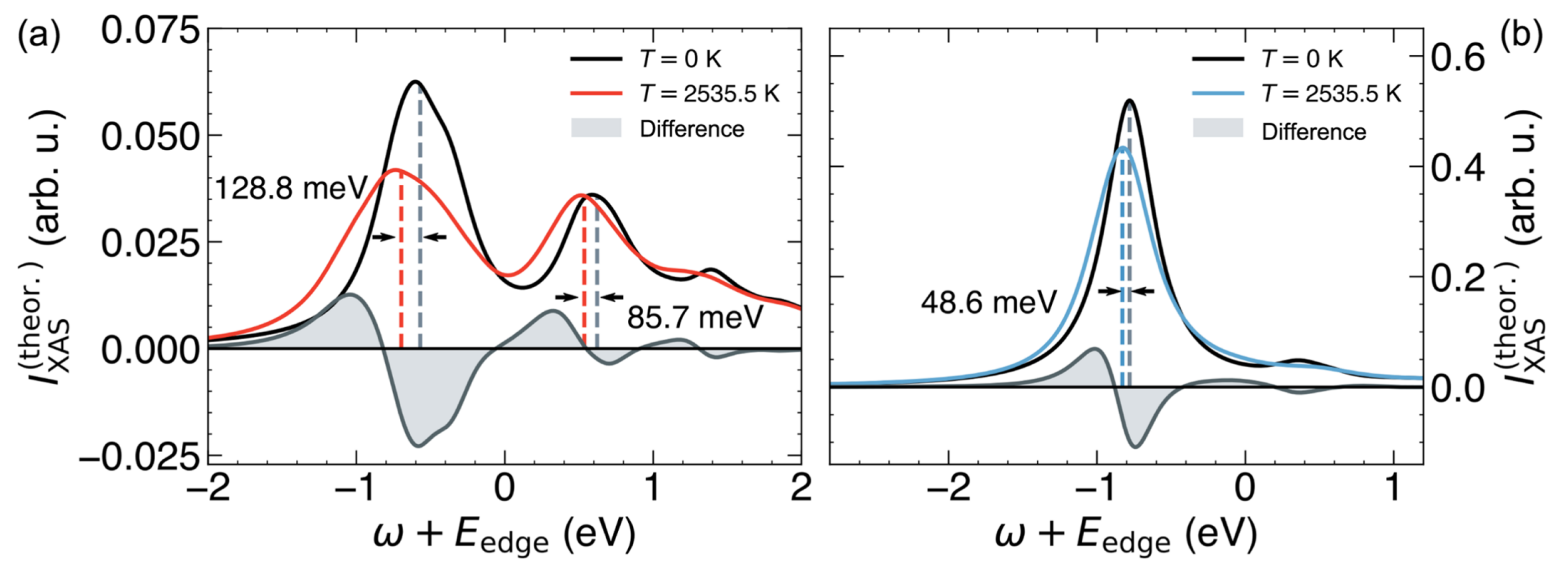

FIG. 6. Theoretical XAS spectra of the three-band Hubbard model at the $\mathrm{O} K$ edge (a) and the Cu $L_{3}$ edge (b) at zero temperature (black) and at $T=2535.5 \mathrm{~K}$ (color).

$$
C_{s}(T)=\gamma T+\beta T^{3},
$$

with $\gamma=2.5 \mathrm{~mJ} \cdot \mathrm{mol}^{-1} \cdot \mathrm{K}^{-2}$ and $\beta=0.25 \mathrm{~mJ} \cdot \mathrm{mol}^{-1} \cdot \mathrm{K}^{-4}$ being the electronic and lattice specific heat coefficients, respectively. Assuming that the pump pulse varies on a timescale shorter than the heating-cooling dynamics, the maximum temperature increase $\Delta T=T_{f}-T_{i}$ is obtained from the absorbed fluence as

$$
Q_{\mathrm{abs}}=\int_{T_{i}}^{T_{f}} C_{s}(T) d T
$$

Given the reflection coefficient of the material $(R=0.15)$ and the estimated penetration depth at $800 \mathrm{~nm}$ $\left(l_{p} \approx 370 \mathrm{~nm}\right)$, we estimate the absorbed energy according to the relation:

$$
Q_{\mathrm{abs}}=\frac{F \cdot A \cdot(1-R)}{2 l_{p}} N_{A} V_{\mathrm{uc}},
$$

where $F=10 \mathrm{~mJ} / \mathrm{cm}^{2}$ denotes the laser fluence, $V_{\text {uc }}=$ $3.787 \times 3.787 \times 13.23 \AA^{3}$ is the volume of the unit cell, and $N_{A}$ is the Avogadro constant. The factor 2 accounts for the presence of two LBCO formula units per unit cell. Setting the initial temperature equal to the base temperature ( $17 \mathrm{~K}$ ), we obtain final temperature $T_{f}=120.3 \mathrm{~K}$. In order to get an estimate for the maximal electronic temperature increase, $\Delta T_{e, \max }=T_{e, \max }-T_{e, 0}$, we make the following approximation of the absorbed fluence:

$$
Q_{\mathrm{abs}}=\int_{T_{e, 0}}^{T_{e, \max }} C_{e}\left(T_{e}\right) d T_{e} .
$$

Here, $C_{e}\left(T_{e}\right)=\gamma T_{e}$ is the electronic (temperaturedependent) specific heat capacity. Using the fluence value estimated from Eq. (C3) and setting the initial $T_{e, 0}$ equal to the base temperature, we obtain a maximum value for $T_{e, \max } \approx 3240 \mathrm{~K}$. However, the pump is attenuated while traveling across the photoexcited volume; hence, a more refined approximation for the maximum electronic temperature increase is [116]

$$
T_{e, \max }=\frac{1}{l_{p}} \int_{0}^{l_{p}} \sqrt{T_{e, 0}^{2}+\frac{2 Q_{\mathrm{abs}}}{\gamma} e^{-z / l_{p}}} d z .
$$

According to Eq. (C5), the maximum electronic temperature increase allowed within our experimental conditions is $T_{e, \max } \approx 2561 \mathrm{~K}$. Having determined the maximum electronic temperature, we examine the corresponding changes in the XAS spectrum. We perform finite-temperature ED calculations within the three-band Hubbard model for temperatures ranging between $T_{e}=0$ and $T_{e}=$ $5300 \mathrm{~K}$. In Fig. 6, we illustrate the effect of electronic heating on the shape of the XAS signal by comparing spectra at $T_{e}=0$ and $T_{e, \max }=2535.5 \mathrm{~K}$. Intuitively, electronic heating creates unoccupied states at lower energies and leads to a shift of the entire absorption spectrum. Both the UHB and ZRS peaks are found to shift. Moreover, the UHB shift at the $\mathrm{Cu} L_{3}$ edge is almost half the one occurring at the $\mathrm{O} K$ edge. This is at odds with the experimental findings in Fig. 2, where the ZRS transition does not shift within experimental accuracy and the UHB shift at the $\mathrm{Cu} L_{3}$ edge is almost twice larger than the one at the $\mathrm{O} K$ edge. This discrepancy strongly suggests that the observed XAS spectral reshaping cannot be explained in terms of a heating-only scenario.

\section{APPENDIX D: FIELD DEPENDENCE OF THE UPPER HUBBARD BAND SHIFT}

In this section, we compare experimental shifts of the UHB with the theoretically expected fluence-dependent 

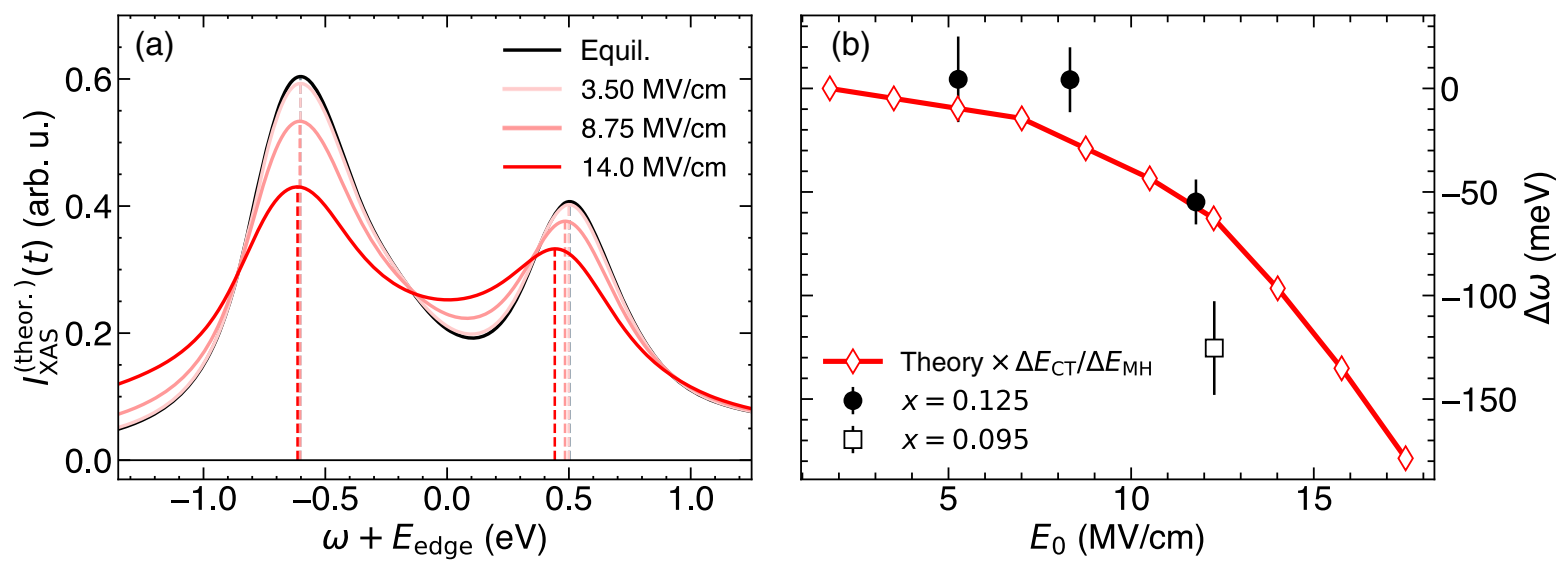

FIG. 7. (a) Theoretical trXAS spectra of the single-band Hubbard model at equilibrium ( $\Delta t=-75 \mathrm{fs}$, black) and at $\Delta t=0 \mathrm{fs}$ for selected pump electric fields. (b) Theoretical UHB peak shift $\Delta \omega^{\text {theor }}=\omega(0 \mathrm{fs})-\omega(-75 \mathrm{fs})$ (red diamonds) as a function of the pump peak field $E_{0}$ and rescaled by the ratio between CT gap and Mott-Hubbard gap. $\Delta E_{\mathrm{CT}}=1.77 \mathrm{eV}$ is the experimental separation of the equilibrium ZRS and UHB bands at the $\mathrm{O} K$ edge, while $\Delta E_{\mathrm{MH}}=1.03 \mathrm{eV}$ is the separation between LHB and UHB in the single-band Hubbard model. Experimental UHB peak shift values at the $\mathrm{Cu} L_{3}$ edge for the two dopings are indicated by black symbols.

trXAS spectra of a single-band Hubbard model. The trXAS calculations are performed on a 12D Betts cluster with two holes [in order to preserve $\mathrm{SU}(2)$ symmetry] and for variable peak electric fields between $E_{0}=1.75 \mathrm{MV} / \mathrm{cm}$ and $E_{0}=17.5 \mathrm{MV} / \mathrm{cm}$. The spectra, reported in Fig. 7(a), are calculated by using the same interaction parameters considered in Fig. 5, and the theoretical peak positions in Fig. 7(b) are defined as the energy of the peak maximum. While the UHB is found to shift appreciably, the LHB shift is much less pronounced and discernible only for the highest field amplitudes considered here. In particular, the peak shift is nonlinear in the field amplitude, as opposed to the predicted Hubbard $U$ reduction by dynamical screening reported in Ref. [21]. Experimental data for the UHB peak shift at the $\mathrm{Cu} L_{3}$ edge are also shown in Fig. 7(b) for two compositions: $x=0.095$ [from Fig. 2(b)] and $x=0.125$ (see Supplemental Material [51], Sec. IV). The experimental and theoretical trends (when rescaled by the relative size of the Mott/CT gap) agree on a quantitative level and outline a nonlinear field dependence of the pumpinduced Hubbard $U$ reduction.

[1] J. Hubbard, Electron Correlations in Narrow Energy Bands, Proc. R. Soc. A 276, 238 (1963).

[2] M. Imada, A. Fujimori, and Y. Tokura, Metal-Insulator Transitions, Rev. Mod. Phys. 70, 1039 (1998).

[3] D. Basov, R. Averitt, and D. Hsieh, Towards Properties on Demand in Quantum Materials, Nat. Mater. 16, 1077 (2017).

[4] Y. Tokura, M. Kawasaki, and N. Nagaosa, Emergent Functions of Quantum Materials, Nat. Phys. 13, 1056 (2017).
[5] F. Novelli, D. Fausti, F. Giusti, F. Parmigiani, and M. Hoffmann, Mixed Regime of Light-Matter Interaction Revealed by Phase Sensitive Measurements of the Dynamical Franz-Keldysh Effect, Sci. Rep. 3, 1227 (2013).

[6] M. Schultze, E. M. Bothschafter, A. Sommer, S. Holzner, W. Schweinberger, M. Fiess, M. Hofstetter, R. Kienberger, V. Apalkov, V. S. Yakovlev, M. I. Stockman, and F. Krausz, Controlling Dielectrics with the Electric Field of Light, Nature (London) 493, 75 (2013).

[7] M. Schultze, K. Ramasesha, C. Pemmaraju, S. Sato, D. Whitmore, A. Gandman, J. S. Prell, L. J. Borja, D. Prendergast, K. Yabana, D. M. Neumark, and S. R. Leone, Attosecond Band-Gap Dynamics in Silicon, Science 346, 1348 (2014).

[8] M. Lucchini, S. A. Sato, A. Ludwig, J. Herrmann, M. Volkov, L. Kasmi, Y. Shinohara, K. Yabana, L. Gallmann, and U. Keller, Attosecond Dynamical Franz-Keldysh Effect in Polycrystalline Diamond, Science 353, 916 (2016).

[9] F. Schlaepfer, M. Lucchini, S. A. Sato, M. Volkov, L. Kasmi, N. Hartmann, A. Rubio, L. Gallmann, and U. Keller, Attosecond Optical-Field-Enhanced Carrier Injection into the GaAs Conduction Band, Nat. Phys. 14, 560 (2018).

[10] O. Grånäs et al., Ultrafast Modification of the Electronic Structure of a Correlated Insulator, arXiv:2008.11115.

[11] M. Först, C. Manzoni, S. Kaiser, Y. Tomioka, Y. Tokura, R. Merlin, and A. Cavalleri, Nonlinear Phononics as an Ultrafast Route to Lattice Control, Nat. Phys. 7, 854 (2011).

[12] A. Subedi, A. Cavalleri, and A. Georges, Theory of Nonlinear Phononics for Coherent Light Control of Solids, Phys. Rev. B 89, 220301(R) (2014).

[13] R. Mankowsky, A. Subedi, M. Först, S. O. Mariager, M. Chollet, H. T. Lemke, J. S. Robinson, J. M. Glownia, M. P. Minitti, A. Frano, M. Fechner, N. A. Spaldin, T. Loew, B. Keimer, A. Georges, and A. Cavalleri, Nonlinear Lattice 
Dynamics as a Basis for Enhanced Superconductivity in $\mathrm{YBa}_{2} \mathrm{Cu}_{3} \mathrm{O}_{6.5}$, Nature (London) 516, 71 (2014).

[14] Y. H. Wang, H. Steinberg, P. Jarillo-Herrero, and N. Gedik, Observation of Floquet-Bloch States on the Surface of a Topological Insulator, Science 342, 453 (2013).

[15] F. Mahmood, C.-K. Chan, Z. Alpichshev, D. Gardner, Y. Lee, P. A. Lee, and N. Gedik, Selective Scattering between Floquet-Bloch and Volkov States in a Topological Insulator, Nat. Phys. 12, 306 (2016).

[16] J. W. McIver, B. Schulte, F.-U. Stein, T. Matsuyama, G. Jotzu, G. Meier, and A. Cavalleri, Light-Induced Anomalous Hall Effect in Graphene, Nat. Phys. 16, 38 (2020).

[17] S. Kaiser, S. R. Clark, D. Nicoletti, G. Cotugno, R. I. Tobey, N. Dean, S. Lupi, H. Okamoto, T. Hasegawa, D. Jaksch, and A. Cavalleri, Optical Properties of a Vibrationally Modulated Solid State Mott Insulator, Sci. Rep. 4, 3823 (2014).

[18] R. Singla, G. Cotugno, S. Kaiser, M. Först, M. Mitrano, H. Y. Liu, A. Cartella, C. Manzoni, H. Okamoto, T. Hasegawa, S. R. Clark, D. Jaksch, and A. Cavalleri, THz-Frequency Modulation of the Hubbard $U$ in an Organic Mott Insulator, Phys. Rev. Lett. 115, 187401 (2015).

[19] M. Buzzi et al., Photomolecular High-Temperature Superconductivity, Phys. Rev. X 10, 031028 (2020).

[20] Y. Kawakami, S. Iwai, T. Fukatsu, M. Miura, N. Yoneyama, T. Sasaki, and N. Kobayashi, Optical Modulation of Effective On-Site Coulomb Energy for the Mott Transition in an Organic Dimer Insulator, Phys. Rev. Lett. 103, 066403 (2009).

[21] N. Tancogne-Dejean, M. A. Sentef, and A. Rubio, Ultrafast Modification of Hubbard $U$ in a Strongly Correlated Material: Ab initio High-Harmonic Generation in $\mathrm{NiO}$, Phys. Rev. Lett. 121, 097402 (2018).

[22] G. E. Topp, N. Tancogne-Dejean, A. F. Kemper, A. Rubio, and M. A. Sentef, All-Optical Nonequilibrium Pathway to Stabilising Magnetic Weyl Semimetals in Pyrochlore Iridates, Nat. Commun. 9, 4452 (2018).

[23] D. Golež, M. Eckstein, and P. Werner, Multiband Nonequilibrium $G W+$ EDMFT Formalism for Correlated Insulators, Phys. Rev. B 100, 235117 (2019).

[24] N. Tancogne-Dejean, M. A. Sentef, and A. Rubio, Ultrafast Transient Absorption Spectroscopy of the ChargeTransfer Insulator NiO: Beyond the Dynamical FranzKeldysh Effect, Phys. Rev. B 102, 115106 (2020).

[25] S. Beaulieu, S. Dong, N. Tancogne-Dejean, M. Dendzik, T. Pincelli, J. Maklar, R. P. Xian, M. A. Sentef, M. Wolf, A. Rubio, L. Rettig, and R. Ernstorfer, Ultrafast Dynamical Lifshitz Transition, Sci. Adv. 7, eabd9275 (2021).

[26] V. N. Valmispild, C. Dutreix, M. Eckstein, M. I. Katsnelson, A.I. Lichtenstein, and E. A. Stepanov, Dynamically Induced Doublon Repulsion in the FermiHubbard Model Probed by a Single-Particle Density of States, Phys. Rev. B 102, 220301(R) (2020).

[27] J. Zhang and R. Averitt, Dynamics and Control in Complex Transition Metal Oxides, Annu. Rev. Mater. Res. 44, 19 (2014).

[28] C. Ahn, A. Cavalleri, A. Georges, S. Ismail-Beigi, A. J. Millis, and J.-M. Triscone, Designing and Controlling the
Properties of Transition Metal Oxide Quantum Materials, Nat. Mater. 20, 1462 (2021).

[29] P. A. Lee, N. Nagaosa, and X.-G. Wen, Doping a Mott Insulator: Physics of High-Temperature Superconductivity, Rev. Mod. Phys. 78, 17 (2006).

[30] B. Keimer, S. A. Kivelson, M. R. Norman, S. Uchida, and J. Zaanen, From Quantum Matter to High-Temperature Superconductivity in Copper Oxides, Nature (London) 518, 179 (2015).

[31] A. Georges, G. Kotliar, W. Krauth, and M. J. Rozenberg, Dynamical Mean-Field Theory of Strongly Correlated Fermion Systems and the Limit of Infinite Dimensions, Rev. Mod. Phys. 68, 13 (1996).

[32] A. Damascelli, Z. Hussain, and Z.-X. Shen, AngleResolved Photoemission Studies of the Cuprate Superconductors, Rev. Mod. Phys. 75, 473 (2003).

[33] R. Rossi, F. Šimkovic, and M. Ferrero, Renormalized Perturbation Theory at Large Expansion Orders, Europhys. Lett. 132, 11001 (2020).

[34] D. J. Scalapino, A Common Thread: The Pairing Interaction for Unconventional Superconductors, Rev. Mod. Phys. 84, 1383 (2012).

[35] F. Nilsson, K. Karlsson, and F. Aryasetiawan, Dynamically Screened Coulomb Interaction in the Parent Compounds of Hole-Doped Cuprates: Trends and Exceptions, Phys. Rev. B 99, 075135 (2019).

[36] P. Abbamonte, A. Rusydi, S. Smadici, G. D. Gu, G. A. Sawatzky, and D. L. Feng, Spatially modulated 'Mottness' in $\mathrm{La}_{2-x} \mathrm{Ba}_{x} \mathrm{CuO}_{4}$, Nat. Phys. 1, 155 (2005).

[37] J. M. Tranquada, H. Woo, T. G. Perring, H. Goka, G. D. Gu, G. Xu, M. Fujita, and K. Yamada, Quantum Magnetic Excitations from Stripes in Copper Oxide Superconductors, Nature (London) 429, 534 (2004).

[38] M. Hücker, M. v. Zimmermann, G. D. Gu, Z. J. Xu, J. S. Wen, G. Xu, H. J. Kang, A. Zheludev, and J. M. Tranquada, Stripe Order in Superconducting $\mathrm{La}_{2-x} \mathrm{Ba}_{x} \mathrm{CuO}_{4}(0.095 \leqslant$ $x \leqslant 0.155)$, Phys. Rev. B 83, 104506 (2011).

[39] A. Cavalleri, M. Rini, H. H. W. Chong, S. Fourmaux, T. E. Glover, P. A. Heimann, J. C. Kieffer, and R. W. Schoenlein, Band-Selective Measurements of Electron Dynamics in $\mathrm{VO}_{2}$ Using Femtosecond Near-Edge X-Ray Absorption, Phys. Rev. Lett. 95, 067405 (2005).

[40] C. Stamm, T. Kachel, N. Pontius, R. Mitzner, T. Quast, K. Holldack, S. Khan, C. Lupulescu, E. F. Aziz, M. Wietstruk, H. A. Dürr, and W. Eberhardt, Femtosecond Modification of Electron Localization and Transfer of Angular Momentum in Nickel, Nat. Mater. 6, 740 (2007).

[41] A. Moulet, J. B. Bertrand, T. Klostermann, A. Guggenmos, N. Karpowicz, and E. Goulielmakis, Soft X-Ray Excitonics, Science 357, 1134 (2017).

[42] L. M. Carneiro, S. K. Cushing, C. Liu, Y. Su, P. Yang, A. P. Alivisatos, and S. R. Leone, Excitation-wavelength-dependent small polaron trapping of photoexcited carriers in $\alpha-\mathrm{Fe}_{2} \mathrm{O}_{3}$, Nat. Mater. 16, 819 (2017).

[43] M. Zürch, H.-T. Chang, L. J. Borja, P. M. Kraus, S. K. Cushing, A. Gandman, C. J. Kaplan, M. H. Oh, J. S. Prell, D. Prendergast, C. D. Pemmaraju, D. M. Neumark, and S. R. Leone, Direct and Simultaneous Observation of Ultrafast Electron and Hole Dynamics in Germanium, Nat. Commun. 8, 15734 (2017). 
[44] A. R. Attar, H.-T. Chang, A. Britz, X. Zhang, M.-F. Lin, A. Krishnamoorthy, T. Linker, D. Fritz, D. M. Neumark, R. K. Kalia, A. Nakano, P. Ajayan, P. Vashishta, U. Bergmann, and S. R. Leone, Simultaneous Observation of CarrierSpecific Redistribution and Coherent Lattice Dynamics in $2 \mathrm{H}-\mathrm{MoTe}_{2}$ with Femtosecond Core-Level Spectroscopy, ACS Nano 14, 15829 (2020).

[45] A. Britz, A. R. Attar, X. Zhang, H.-T. Chang, C. Nyby, A. Krishnamoorthy, S. H. Park, S. Kwon, M. Kim, D. Nordlund, S. Sainio, T. F. Heinz, S. R. Leone, A. M. Lindenberg, A. Nakano, P. Ajayan, P. Vashishta, D. Fritz, M.-F. Lin, and U. Bergmann, Carrier-Specific Dynamics in 2H-MoTe 2 Observed by Femtosecond Soft X-Ray Absorption Spectroscopy Using an X-Ray Free-Electron Laser, Struct. Dyn. 8, 014501 (2021).

[46] J. R. L. Mardegan et al., Ultrafast Electron Localization in the $\mathrm{EuNi}_{2}\left(\mathrm{Si}_{0.21} \mathrm{Ge}_{0.79}\right)_{2}$ Correlated Metal, Phys. Rev. Research 3, 033211 (2021).

[47] H. Jang et al., Time-Resolved Resonant Elastic Soft X-Ray Scattering at Pohang Accelerator Laboratory X-Ray Free Electron Laser, Rev. Sci. Instrum. 91, 083904 (2020).

[48] M. Ogata and H. Fukuyama, The $t-J$ Model for the Oxide High- $T_{c}$ Superconductors, Rep. Prog. Phys. 71, 036501 (2008).

[49] F. C. Zhang and T. M. Rice, Effective Hamiltonian for the Superconducting $\mathrm{Cu}$ Oxides, Phys. Rev. B 37, 3759 (1988).

[50] H. Eskes, M. B. J. Meinders, and G. A. Sawatzky, Anomalous Transfer of Spectral Weight in Doped Strongly Correlated Systems, Phys. Rev. Lett. 67, 1035 (1991).

[51] See Supplemental Material at http://link.aps.org/ supplemental/10.1103/PhysRevX.12.011013 for details on the experimental data analysis, the analysis of the time-dependent XAS spectra, the theoretical XAS calculations within the three-band Hubbard model for the underdoped $(x=12.5 \%)$ as well as the half-filling cases, the complete analysis of the temperature-dependence of the XAS spectra, as well as complementary experimental data pertaining to a LBCO compound with $x=12.5 \%$ doping.

[52] C. T. Chen, F. Sette, Y. Ma, M. S. Hybertsen, E. B. Stechel, W. M. C. Foulkes, M. Schulter, S.-W. Cheong, A. S. Cooper, L. W. Rupp, B. Batlogg, Y. L. Soo, Z. H. Ming, A. Krol, and Y.H. Kao, Electronic States in $\mathrm{La}_{2-x} \mathrm{Sr}_{x} \mathrm{CuO}_{4+\delta}$ Probed by Soft-X-Ray Absorption, Phys. Rev. Lett. 66, 104 (1991).

[53] C. T. Chen, L. H. Tjeng, J. Kwo, H. L. Kao, P. Rudolf, F. Sette, and R. M. Fleming, Out-of-plane orbital characters of intrinsic and doped holes in $\mathrm{La}_{2-x} \mathrm{Sr}_{x} \mathrm{CuO}_{4}$, Phys. Rev. Lett. 68, 2543 (1992).

[54] N. Nücker, E. Pellegrin, P. Schweiss, J. Fink, S. L. Molodtsov, C. T. Simmons, G. Kaindl, W. Frentrup, A. Erb, and G. Müller-Vogt, Site-Specific and DopingDependent Electronic Structure of $\mathrm{YBa}_{2} \mathrm{Cu}_{3} \mathrm{O}_{x}$ Probed by $\mathrm{O} 1 s$ and $\mathrm{Cu} 2 p$ X-Ray-Absorption Spectroscopy, Phys. Rev. B 51, 8529 (1995).

[55] V. V. Baranov and V. V. Kabanov, Theory of Electronic Relaxation in a Metal Excited by an Ultrashort Optical Pump, Phys. Rev. B 89, 125102 (2014).
[56] H. Okamoto, T. Miyagoe, K. Kobayashi, H. Uemura, H. Nishioka, H. Matsuzaki, A. Sawa, and Y. Tokura, Ultrafast Charge Dynamics in Photoexcited $\mathrm{Nd}_{2} \mathrm{CuO}_{4}$ and $\mathrm{La}_{2} \mathrm{CuO}_{4}$ Cuprate Compounds Investigated by Femtosecond Absorption Spectroscopy, Phys. Rev. B 82, 060513(R) (2010).

[57] H. Okamoto, T. Miyagoe, K. Kobayashi, H. Uemura, H. Nishioka, H. Matsuzaki, A. Sawa, and Y. Tokura, Photoinduced Transition from Mott Insulator to Metal in the Undoped Cuprates $\mathrm{Nd}_{2} \mathrm{CuO}_{4}$ and $\mathrm{La}_{2} \mathrm{CuO}_{4}$, Phys. Rev. B 83, 125102 (2011).

[58] S. Dal Conte et al., Snapshots of the Retarded Interaction of Charge Carriers with Ultrafast Fluctuations in Cuprates, Nat. Phys. 11, 421 (2015).

[59] F. Carbone, D.-S. Yang, E. Giannini, and A. H. Zewail, Direct Role of Structural Dynamics in Electron-Lattice Coupling of Superconducting Cuprates, Proc. Natl. Acad. Sci. U.S.A. 105, 20161 (2008).

[60] T. Konstantinova, J. D. Rameau, A.H. Reid, O. Abdurazakov, L. Wu, R. Li, X. Shen, G. Gu, Y. Huang, L. Rettig, I. Avigo, M. Ligges, J. K. Freericks, A. F. Kemper, H. A. Dürr, U. Bovensiepen, P. D. Johnson, X. Wang, and Y. Zhu, Nonequilibrium Electron and Lattice Dynamics of Strongly Correlated $\mathrm{Bi}_{2} \mathrm{Sr}_{2} \mathrm{CaCu}_{2} \mathrm{O}_{8+\delta}$ Single Crystals, Sci. Adv. 4, eaap7427 (2018).

[61] L. Perfetti, P. A. Loukakos, M. Lisowski, U. Bovensiepen, H. Eisaki, and M. Wolf, Ultrafast Electron Relaxation in Superconducting $\mathrm{Bi}_{2} \mathrm{Sr}_{2} \mathrm{CaCu}_{2} \mathrm{O}_{8+\delta}$ by Time-Resolved Photoelectron Spectroscopy, Phys. Rev. Lett. 99, 197001 (2007).

[62] C. L. Smallwood, J. P. Hinton, C. Jozwiak, W. Zhang, J. D. Koralek, H. Eisaki, D.-H. Lee, J. Orenstein, and A. Lanzara, Tracking Cooper Pairs in a Cuprate Superconductor by Ultrafast Angle-Resolved Photoemission, Science 336, 1137 (2012).

[63] G. L. Dakovski, T. Durakiewicz, J.-X. Zhu, P.S. Riseborough, G. Gu, S. M. Gilbertson, A. Taylor, and G. Rodriguez, Quasiparticle Dynamics across the Full Brillouin Zone of $\mathrm{Bi}_{2} \mathrm{Sr}_{2} \mathrm{CaCu}_{2} \mathrm{O}_{8+\delta}$ Traced with Ultrafast Time and Angle-Resolved Photoemission Spectroscopy, Struct. Dyn. 2, 054501 (2015).

[64] J. D. Rameau, S. Freutel, A. F. Kemper, M. A. Sentef, J. K. Freericks, I. Avigo, M. Ligges, L. Rettig, Y. Yoshida, H. Eisaki, J. Schneeloch, R. D. Zhong, Z. J. Xu, G. D. Gu, P. D. Johnson, and U. Bovensiepen, Energy Dissipation from a Correlated System Driven Out of Equilibrium, Nat. Commun. 7, 13761 (2016).

[65] F. Boschini, E. H. da Silva Neto, E. Razzoli, M. Zonno, S. Peli, R. P. Day, M. Michiardi, M. Schneider, B. Zwartsenberg, P. Nigge, R. D. Zhong, J. Schneeloch, G. D. Gu, S. Zhdanovich, A. K. Mills, G. Levy, D. J. Jones, C. Giannetti, and A. Damascelli, Collapse of Superconductivity in Cuprates via Ultrafast Quenching of Phase Coherence, Nat. Mater. 17, 416 (2018).

[66] Z. Lenarčič and P. Prelovšek, Ultrafast Charge Recombination in a Photoexcited Mott-Hubbard Insulator, Phys. Rev. Lett. 111, 016401 (2013).

[67] F. Novelli, G. De Filippis, V. Cataudella, M. Esposito, I. Vergara, F. Cilento, E. Sindici, A. Amaricci, C. Giannetti, D. Prabhakaran, S. Wall, A. Perucchi, S. Dal Conte, 
G. Cerullo, M. Capone, A. Mishchenko, M. Grüninger, N. Nagaosa, F. Parmigiani, and D. Fausti, Witnessing the Formation and Relaxation of Dressed Quasi-Particles in a Strongly Correlated Electron System, Nat. Commun. 5, 5112 (2014).

[68] F. Cilento, G. Manzoni, A. Sterzi, S. Peli, A. Ronchi, A. Crepaldi, F. Boschini, C. Cacho, R. Chapman, E. Springate, H. Eisaki, M. Greven, M. Berciu, A. F. Kemper, A. Damascelli, M. Capone, C. Giannetti, and F. Parmigiani, Dynamics of Correlation-Frozen Antinodal Quasiparticles in Superconducting Cuprates, Sci. Adv. 4, aar1998 (2018).

[69] S. Wall, D. Brida, S. Clark, H. P. Ehrke, D. Jaksch, A. Ardavan, S. Bonora, H. Uemura, Y. Takahashi, T. Hasegawa, H. Okamoto, G. Cerullo, and A. Cavalleri, Quantum Interference between Charge Excitation Paths in a Solid-State Mott Insulator, Nat. Phys. 7, 114 (2011).

[70] M. Mitrano, G. Cotugno, S. R. Clark, R. Singla, S. Kaiser, J. Stähler, R. Beyer, M. Dressel, L. Baldassarre, D. Nicoletti, A. Perucchi, T. Hasegawa, H. Okamoto, D. Jaksch, and A. Cavalleri, Pressure-Dependent Relaxation in the Photoexcited Mott Insulator ET - $\mathrm{F}_{2}$ TCNQ: Influence of Hopping and Correlations on Quasiparticle Recombination Rates, Phys. Rev. Lett. 112, 117801 (2014).

[71] N. Strohmaier, D. Greif, R. Jördens, L. Tarruell, H. Moritz, T. Esslinger, R. Sensarma, D. Pekker, E. Altman, and E. Demler, Observation of Elastic Doublon Decay in the Fermi-Hubbard Model, Phys. Rev. Lett. 104, 080401 (2010).

[72] R. Sensarma, D. Pekker, E. Altman, E. Demler, N. Strohmaier, D. Greif, R. Jördens, L. Tarruell, H. Moritz, and T. Esslinger, Lifetime of Double Occupancies in the Fermi-Hubbard Model, Phys. Rev. B 82, 224302 (2010).

[73] Z. Lenarčič and P. Prelovšek, Charge Recombination in Undoped Cuprates, Phys. Rev. B 90, 235136 (2014).

[74] D. J. Higley, A. H. Reid, Z. Chen, L. L. Guyader, O. Hellwig, A. A. Lutman, T. Liu, P. Shafer, T. Chase, G. L. Dakovski, A. Mitra, E. Yuan, J. Schlappa, H. A. Dürr, W. F. Schlotter, and J. Stöhr, Femtosecond X-Ray Induced Changes of the Electronic and Magnetic Response of Solids from Electron Redistribution, Nat. Commun. 10, 5289 (2019).

[75] B. Mansart, M. J. G. Cottet, G. F. Mancini, T. Jarlborg, S. B. Dugdale, S. L. Johnson, S. O. Mariager, C. J. Milne, P. Beaud, S. Grübel, J. A. Johnson, T. Kubacka, G. Ingold, K. Prsa, H. M. Rønnow, K. Conder, E. Pomjakushina, M. Chergui, and F. Carbone, Temperature-Dependent Electron-Phonon Coupling in $\mathrm{La}_{2-x} \mathrm{Sr}_{x} \mathrm{CuO}_{4}$ Probed by Femtosecond X-Ray Diffraction, Phys. Rev. B 88, 054507 (2013).

[76] G. D. Mahan, Many-Particle Physics, 3rd ed. (Plenum, New York, 2000)

[77] M. S. Hybertsen, E. B. Stechel, W. M. C. Foulkes, and M. Schlüter, Model for Low-Energy Electronic States Probed by X-Ray Absorption in High-T $T_{c}$ Cuprates, Phys. Rev. B 45, 10032 (1992).

[78] L. J. P. Ament, Resonant Inelastic X-Ray Scattering Studies of Elementary Excitations, Ph. D. thesis, Leiden University, 2010.
[79] L. F. Mattheiss, Electronic Band Properties and Superconductivity in $\mathrm{La}_{2-y} \mathrm{X}_{y} \mathrm{CuO}_{4}$, Phys. Rev. Lett. 58, 1028 (1987).

[80] V. J. Emery, Theory of High- $T_{c}$ Superconductivity in Oxides, Phys. Rev. Lett. 58, 2794 (1987).

[81] C. M. Varma, S. Schmitt-Rink, and E. Abrahams, Charge Transfer Excitations and Superconductivity in "Ionic" Metals, Solid State Commun. 62, 681 (1987).

[82] C.-C. Chen, M. Sentef, Y. F. Kung, C. J. Jia, R. Thomale, B. Moritz, A.P. Kampf, and T. P. Devereaux, Doping Evolution of the Oxygen K-Edge X-Ray Absorption Spectra of Cuprate Superconductors Using a Three-Orbital Hubbard Model, Phys. Rev. B 87, 165144 (2013).

[83] C. Nicolas and C. Miron, Lifetime Broadening of CoreExcited and-Ionized States, J. Electron Spectrosc. Relat. Phenom. 185, 267 (2012), special issue in honor of Prof. T. Darrah Thomas: High-Resolution Spectroscopy of Isolated Species.

[84] M. Rossi, R. Arpaia, R. Fumagalli, M. M. Sala, D. Betto, K. Kummer, G. M. De Luca, J. van den Brink, M. Salluzzo, N. B. Brookes, L. Braicovich, and G. Ghiringhelli, Experimental Determination of Momentum-Resolved Electron-Phonon Coupling, Phys. Rev. Lett. 123, 027001 (2019).

[85] S. Maekawa, T. Tohyama, S. E. Barnes, S. Ishihara, W. Koshibae, and G. Khaliullin, Physics of Transition Metal Oxides, 1st ed. (Springer, Berlin, 2004).

[86] Y. F. Kung, C.-C. Chen, Y. Wang, E. W. Huang, E. A. Nowadnick, B. Moritz, R. T. Scalettar, S. Johnston, and T. P. Devereaux, Characterizing the Three-Orbital Hubbard Model with Determinant Quantum Monte Carlo, Phys. Rev. B 93, 155166 (2016).

[87] K. Tsutsui and T. Tohyama, Incident-Energy-Dependent Spectral Weight of Resonant Inelastic X-Ray Scattering in Doped Cuprates, Phys. Rev. B 94, 085144 (2016).

[88] K. Tsutsui, K. Shinjo, and T. Tohyama, Antiphase Oscillations in the Time-Resolved Spin Structure Factor of a Photoexcited Mott Insulator, Phys. Rev. Lett. 126, 127404 (2021).

[89] J. Duris et al., Tunable Isolated Attosecond X-Ray Pulses with Gigawatt Peak Power from a Free-Electron Laser, Nat. Photonics 14, 30 (2020).

[90] B. Buades, A. Picón, E. Berger, I. León, N. Di Palo, S. L. Cousin, C. Cocchi, E. Pellegrin, J. H. Martin, S. MañasValero, E. Coronado, T. Danz, C. Draxl, M. Uemoto, K. Yabana, M. Schultze, S. Wall, M. Zürch, and J. Biegert, Attosecond State-Resolved Carrier Motion in Quantum Materials Probed by Soft X-Ray XANES, Appl. Phys. Rev. 8, 011408 (2021).

[91] W. Hu, S. Kaiser, D. Nicoletti, C. R. Hunt, I. Gierz, M. C. Hoffmann, M. Le Tacon, T. Loew, B. Keimer, and A. Cavalleri, Optically Enhanced Coherent Transport in $\mathrm{YBa}_{2} \mathrm{Cu}_{3} \mathrm{O}_{6.5}$ by Ultrafast Redistribution of Interlayer Coupling, Nat. Mater. 13, 705 (2014).

[92] S. Kaiser, C. R. Hunt, D. Nicoletti, W. Hu, I. Gierz, H. Y. Liu, M. Le Tacon, T. Loew, D. Haug, B. Keimer, and A. Cavalleri, Optically Induced Coherent Transport Far above $T_{c}$ in Underdoped $\mathrm{YBa}_{2} \mathrm{Cu}_{3} \mathrm{O}_{6+\delta}$, Phys. Rev. B 89, 184516 (2014). 
[93] D. Nicoletti, E. Casandruc, Y. Laplace, V. Khanna, C. R. Hunt, S. Kaiser, S. S. Dhesi, G. D. Gu, J. P. Hill, and A. Cavalleri, Optically Induced Superconductivity in Striped $\mathrm{La}_{2-x} \mathrm{Ba}_{x} \mathrm{CuO}_{4}$ by Polarization-Selective Excitation in the near Infrared, Phys. Rev. B 90, 100503(R) (2014).

[94] D. Nicoletti, D. Fu, O. Mehio, S. Moore, A. S. Disa, G. D. $\mathrm{Gu}$, and A. Cavalleri, Magnetic-Field Tuning of LightInduced Superconductivity in Striped $\mathrm{La}_{2-x} \mathrm{Ba}_{x} \mathrm{CuO}_{4}$, Phys. Rev. Lett. 121, 267003 (2018).

[95] K. A. Cremin, J. Zhang, C. C. Homes, G. D. Gu, Z. Sun, M. M. Fogler, A. J. Millis, D. N. Basov, and R. D. Averitt, Photoenhanced Metastable c-Axis Electrodynamics in Stripe-Ordered Cuprate $\mathrm{La}_{1.885} \mathrm{Ba}_{0.115} \mathrm{CuO}_{4}$, Proc. Natl. Acad. Sci. U.S.A. 116, 19875 (2019).

[96] A. Ramos-Alvarez, N. Fleischmann, L. Vidas, A. Fernandez-Rodriguez, A. Palau, and S. Wall, Probing the Lattice Anharmonicity of Superconducting $\mathrm{YBa}_{2} \mathrm{Cu}_{3} \mathrm{O}_{7-\delta}$ via Phonon Harmonics, Phys. Rev. B 100, 184302 (2019).

[97] C. Weber, C. Yee, K. Haule, and G. Kotliar, Scaling of the Transition Temperature of Hole-Doped Cuprate Superconductors with the Charge-Transfer Energy, Europhys. Lett. 100, 37001 (2012).

[98] W. Ruan, C. Hu, J. Zhao, P. Cai, Y. Peng, C. Ye, R. Yu, X. Li, Z. Hao, C. Jin, X. Zhou, Z.-Y. Weng, and Y. Wang, Relationship between the Parent Charge Transfer Gap and Maximum Transition Temperature in Cuprates, Sci. Bull. 61, 1826 (2016).

[99] A. T. Rømer, T. A. Maier, A. Kreisel, I. Eremin, P. J. Hirschfeld, and B. M. Andersen, Pairing in the TwoDimensional Hubbard Model from Weak to Strong Coupling, Phys. Rev. Research 2, 013108 (2020).

[100] M. Le Tacon, G. Ghiringhelli, J. Chaloupka, M. M. Sala, V. Hinkov, M. W. Haverkort, M. Minola, M. Bakr, K. J. Zhou, S. Blanco-Canosa, C. Monney, Y. T. Song, G. L. Sun, C. T. Lin, G. M. De Luca, M. Salluzzo, G. Khaliullin, T. Schmitt, L. Braicovich, and B. Keimer, Intense Paramagnon Excitations in a Large Family of High-Temperature Superconductors, Nat. Phys. 7, 725 (2011).

[101] M. Mitrano and Y. Wang, Probing Light-Driven Quantum Materials with Ultrafast Resonant Inelastic X-Ray Scattering, Commun. Phys. 3, 184 (2020).

[102] Y. Wang, Y. Chen, T. P. Devereaux, B. Moritz, and M. Mitrano, X-Ray Scattering from Light-Driven Spin Fluctuations in a Doped Mott Insulator, Commun. Phys. 4, 212 (2021).

[103] H.-Y. Yang, A. M. Läuchli, F. Mila, and K. P. Schmidt, Effective Spin Model for the Spin-Liquid Phase of the
Hubbard Model on the Triangular Lattice, Phys. Rev. Lett. 105, 267204 (2010).

[104] P. Sahebsara and D. Sénéchal, Hubbard Model on the Triangular Lattice: Spiral Order and Spin Liquid, Phys. Rev. Lett. 100, 136402 (2008).

[105] T. Shirakawa, T. Tohyama, J. Kokalj, S. Sota, and S. Yunoki, Ground-State Phase Diagram of the Triangular Lattice Hubbard Model by the Density-Matrix Renormalization Group Method, Phys. Rev. B 96, 205130 (2017).

[106] A. Szasz, J. Motruk, M. P. Zaletel, and J. E. Moore, Chiral Spin Liquid Phase of the Triangular Lattice Hubbard Model: A Density Matrix Renormalization Group Study, Phys. Rev. X 10, 021042 (2020).

[107] H. J. Liao, Z. Y. Xie, J. Chen, Z. Y. Liu, H. D. Xie, R. Z. Huang, B. Normand, and T. Xiang, Gapless Spin-Liquid Ground State in the $S=1 / 2$ Kagome Antiferromagnet, Phys. Rev. Lett. 118, 137202 (2017).

[108] S. Yan, D. A. Huse, and S. R. White, Spin-Liquid Ground State of the $S=1 / 2$ Kagome Heisenberg Antiferromagnet, Science 332, 1173 (2011).

[109] H.-C. Jiang, Z. Wang, and L. Balents, Identifying Topological Order by Entanglement Entropy, Nat. Phys. 8, 902 (2012).

[110] T. Kaneko, T. Shirakawa, S. Sorella, and S. Yunoki, Photoinduced $\eta$ Pairing in the Hubbard Model, Phys. Rev. Lett. 122, 077002 (2019).

[111] F. Peronaci, O. Parcollet, and M. Schiró, Enhancement of Local Pairing Correlations in Periodically Driven Mott Insulators, Phys. Rev. B 101, 161101(R) (2020).

[112] J. Li, D. Golez, P. Werner, and M. Eckstein, $\eta$-Paired Superconducting Hidden Phase in Photodoped Mott Insulators, Phys. Rev. B 102, 165136 (2020).

[113] J. Tindall, F. Schlawin, M. Buzzi, D. Nicoletti, J. R. Coulthard, H. Gao, A. Cavalleri, M. A. Sentef, and D. Jaksch, Dynamical Order and Superconductivity in a Frustrated Many-Body System, Phys. Rev. Lett. 125, 137001 (2020).

[114] S. Zhang, Pseudospin Symmetry and New Collective Modes of the Hubbard Model, Phys. Rev. Lett. 65, 120 (1990).

[115] Y. Wang, Y. Chen, C. Jia, B. Moritz, and T. P. Devereaux, Time-Resolved Resonant Inelastic X-Ray Scattering in a Pumped Mott Insulator, Phys. Rev. B 101, 165126 (2020).

[116] B. Mansart, D. Boschetto, A. Savoia, F. Rullier-Albenque, F. Bouquet, E. Papalazarou, A. Forget, D. Colson, A. Rousse, and M. Marsi, Ultrafast Transient Response and Electron-Phonon Coupling in the Iron-Pnictide Superconductor $\mathrm{Ba}\left(\mathrm{Fe}_{1-x} \mathrm{Co}_{x}\right)_{2} \mathrm{As}_{2}$, Phys. Rev. B 82, 024513 (2010). 